\title{
Article
}

\section{Uniform well-posedness and stability for fractional Navier-Stokes equations with Coriolis force in critical Fourier-Besov-Morrey spaces}

\author{
Azzeddine El Baraka ${ }^{1, *}$ and Mohamed Toumlilin ${ }^{1}$ \\ 1 University Sidi Mohamed Ben Abdellah, FST Fes-Saiss, Laboratory AAFA Department of Mathematics, B.P 2202 \\ Route Immouzer Fes 30000 Morocco.; zzeddine.elbaraka@usmba.ac.ma (A.E.B); mohamed.toumlilin@usmba.ac.ma \\ (M.T) \\ * Correspondence: zzeddine.elbaraka@usmba.ac.ma
}

Received: 09 February 2019; Accepted: 29 May 2019; Published: 30 June 2019.

\begin{abstract}
In this paper, we study the Cauchy problem of the fractional Navier-Stokes equations with Coriolis force in critical Fourier-Besov-Morrey spaces. By using the Fourier localization argument and the Littlewood-Paley theory, we get a local well-posedness results and global well-posedness results with small initial data belonging to the critical Fourier-Besov-Morrey spaces. Moreover; we prove that the corresponding global solution decays to zero as time goes to infinity, and we give the stability result for global solutions.
\end{abstract}

Keywords: Navier-Stokes equations, global well-posedness, Coriolis force, Fourier-Besov-Morrey space.

MSC: 35Q30, 76D05,76D03.

\section{Introduction}

I

$\mathrm{n}$ this paper, we consider the initial value problem of the fractional Navier-Stokes equations with the Coriolis force in $\mathbb{R}^{3}$,

$$
\left\{\begin{array}{l}
u_{t}+\mu(-\Delta)^{\alpha} u+\Omega e_{3} \times u+(u . \nabla) u+\nabla \pi=0 \quad(t, x) \in \mathbb{R}^{+} \times \mathbb{R}^{3}, \\
\nabla \cdot u=0 \\
u(0, x)=u_{0}(x) \quad x \in \mathbb{R}^{3},
\end{array}\right.
$$

where $u=u(t, x)=\left(u_{1}(t, x), u_{2}(t, x), u_{3}(t, x)\right)$ represents the unknown velocity vector, the scalar function $\pi=\pi(t, x)$ denotes the unknown scalar pressure and $u_{0}$ is a divergence free vector field. The constant $\mu>0$ indicates the viscosity coefficient of the fluid, $\Omega \in \mathbb{R}$ represents the speed of rotation around the vertical unit vector $e_{3}=(0,0,1)$, which is called the Coriolis parameter, and $\times$ represents the outer product, hence, $-\Omega e_{3} \times u=\left(\Omega u_{2},-\Omega u_{1}, 0\right)$. We recall that the Coriolis term has an another expression $-\Omega e_{3} \times u=-\Omega \mathrm{J} u$, where the skew-symmetric matrix J defined by

$$
J=\left(\begin{array}{lll}
0 & -1 & 0 \\
1 & 0 & 0 \\
0 & 0 & 0
\end{array}\right)
$$

The operator $(-\Delta)^{\alpha}$ is the Fourier multiplier with symbol $|\xi|^{2 \alpha}$.

When $\alpha=1$, the Equation (1) corresponds to the usual Navier-Stokes equation with Coriolis force, which receives some attention for its importance in geophysical flow applications. In particular, large scale atmospheric and oceanic flows are dominated by rotational effects, see ([1], [2]). When $\alpha=1$ and $\Omega \neq 0$, Hieber and Shibata [3] obtained the uniform global well-posedness for the Navier-Stokes equations with Coriolis force for small initial data in the Sobolev space $H^{\frac{1}{2}}\left(\mathbb{R}^{3}\right)$. Chemin et al. [2,4] established that for any initial data $u_{0}$ belonging to $L^{2}\left(\mathbb{R}^{2}\right)+H^{\frac{1}{2}}\left(\mathbb{R}^{3}\right)$ there exists a unique solution to the Navier-Stokes equations with Coriolis force when $|\Omega|>\Omega_{0}>0$. Iwabuchi and Takada [5] proved the existence of global solutions for 
the Navier-Stokes equations with Coriolis force in Sobolev spaces $\dot{H}^{s}\left(\mathbb{R}^{3}\right)$ with $1 / 2<s<3 / 4$ if the speed of rotation $\Omega$ is large enough compared with the norm of initial data $\left\|u_{0}\right\|_{\dot{H}^{s}}$; they also obtained the global existence and the uniqueness of the mild solution for small initial data in the Fourier-Besov spaces $\mathrm{FB}_{1,2}^{-1}$, and proved the ill-posedness in the space $\mathrm{FB}_{1, q}^{-1}, 2<q \leq \infty$ for all $\Omega \in \mathbb{R}$ (see [6]). For the local existence of solutions of these equations, we quote the results of Giga et al. [7,8] and Sawada [9].

Recently, W. Wang and G. Wu [10] established the global well-posedness of mild solution to the three-dimensional incompressible generalized Navier-Stokes equations with Coriolis force if the initial data are in the Lei-Lin's space $\chi^{1-2 \alpha}$, they also gave Gevrey class regularity of the solution.

In the case $\Omega=0$ and $\alpha=1$, the problem (1) corresponds to the usual Navier-Stokes equations:

$$
\left\{\begin{array}{l}
u_{t}-\mu \Delta u+(u . \nabla) u+\nabla \pi=0 \quad(t, x) \in \mathbb{R}^{+} \times \mathbb{R}^{3}, \\
\nabla \cdot u=0 \\
u(0, x)=u_{0}(x) \quad x \in \mathbb{R}^{3} .
\end{array}\right.
$$

Throughout this paper, we use $\mathcal{F} \dot{\mathcal{N}}_{p, \lambda, q}^{s}$ to denote the homogenous Fourier Besov-Morrey spaces, $C$ will denote constants which can be different at different places, $\mathrm{U} \lesssim \mathrm{V}$ means that there exists a constant $C>0$ such that $\mathrm{U} \leq C \mathrm{~V}$, and $p^{\prime}$ is the conjugate of $p$ satisfying $\frac{1}{p}+\frac{1}{p^{\prime}}=1$ for $1 \leq p \leq \infty$.

Inspired by the works $[6,10-13]$, the aim of this paper is to prove the global existence and the decay property and the stability of the global solutions of the fractional Navier-Stokes equations with Coriolis force (1) in the Fourier-Besov-Morrey space $\mathcal{F} \mathcal{N}_{p, \lambda, q}^{1-2 \alpha+\frac{3}{p^{\prime}}+\frac{\lambda}{p}}\left(\mathbb{R}^{3}\right)$.

\section{Preliminaries and main results}

The results presented in this work are based on homogeneous Littlewood-Paley decomposition in the Fourier variables. We evoke briefly this construction below. We begin by dyadic decomposition of $\mathbb{R}^{n}$.

Choose two nonnegative smooth radial functions $\chi, \varphi$ satisfying

$$
\begin{aligned}
& \operatorname{supp} \varphi \subset\left\{\xi \in \mathbb{R}^{n}: \frac{3}{4} \leq|\xi| \leq \frac{8}{3}\right\}, \quad \sum_{j \in \mathbb{Z}} \varphi\left(2^{-j} \xi\right)=1, \quad \xi \in \mathbb{R}^{n} \backslash\{0\}, \\
& \operatorname{supp} \chi \subset\left\{\xi \in \mathbb{R}^{n}:|\xi| \leq \frac{4}{3}\right\}, \quad \chi(\xi)+\sum_{j \geq 0} \varphi\left(2^{-j} \xi\right)=1, \quad \xi \in \mathbb{R}^{n} .
\end{aligned}
$$

We denote $\varphi_{j}(\xi)=\varphi\left(2^{-j} \xi\right)$ and $\mathcal{P}$ the set of all polynomials. The space of tempered distributions is denoted by $S^{\prime}$. The homogeneous dyadic blocks $\dot{\Delta}_{j}$ and $\dot{S}_{j}$ are defined for all $j \in \mathbb{Z}$ by

$$
\begin{gathered}
\dot{\Delta}_{j} u=\varphi\left(2^{-j} D\right) u=2^{j n} \int h\left(2^{j} y\right) u(x-y) d y, \\
\dot{S}_{j} u=\sum_{k \leq j-1} \dot{\Delta}_{k} u=\chi\left(2^{-j} D\right) u=2^{j n} \int \tilde{h}\left(2^{j} y\right) u(x-y) d y,
\end{gathered}
$$

where $h=\mathcal{F}^{-1} \varphi$ and $\tilde{h}=\mathcal{F}^{-1} \chi$.

First, we give the definition of the Morrey spaces which are a complement to the $L^{p}$ spaces.

Definition 1 ([14,15]). For $1 \leq p<\infty, 0 \leq \lambda<n$, the Morrey spaces $\mathrm{M}_{p}^{\lambda}=\mathrm{M}_{p}^{\lambda}\left(\mathbb{R}^{n}\right)$ is defined as the set of functions $f \in L_{l o c}^{p}\left(\mathbb{R}^{n}\right)$ such that

$$
\|f\|_{\mathbf{M}_{p}^{\lambda}}=\sup _{x_{0} \in \mathbb{R}^{n}} \sup _{r>0} r^{-\frac{\lambda}{p}}\|f\|_{L^{p}\left(B\left(x_{0}, r\right)\right)}<\infty,
$$

where $B\left(x_{0}, r\right)$ denotes the ball in $\mathbb{R}^{n}$ with center $x_{0}$ and radius $r$. 
It is easy to observe that the relation $\mathrm{M}_{p_{1}}^{\lambda} \hookrightarrow \mathrm{M}_{p_{2}}^{\mu}$ provided $\frac{n-\mu}{p_{2}} \geq \frac{n-\lambda}{p_{1}}$ and $p_{2} \leq p_{1}$, and $\mathrm{M}_{p}^{0}=L^{p}$.

If $1 \leq p_{1}, p_{2}, p_{3}<\infty$ and $0 \leq \lambda_{1}, \lambda_{2}, \lambda_{3}<n$ with $\frac{1}{p_{3}}=\frac{1}{p_{1}}+\frac{1}{p_{2}}$ and $\frac{\lambda_{3}}{p_{3}}=\frac{\lambda_{1}}{p_{1}}+\frac{\lambda_{2}}{p_{2}}$, then we have the Hölder inequality

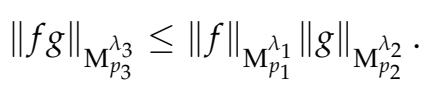

Also, for $1 \leq p<\infty$ and $0 \leq \lambda<n$,

$$
\|\varphi * g\|_{\mathrm{M}_{p}^{\lambda}} \leq\|\varphi\|_{L^{1}}\|g\|_{\mathrm{M}_{p}^{\lambda}}
$$

for all $\varphi \in L^{1}$ and $g \in \mathbf{M}_{p}^{\lambda}$.

Definition 2. (Homogeneous Besov-Morrey spaces ) Let $s \in \mathbb{R}, 1 \leq p<+\infty, 1 \leq q \leq+\infty$, and $0 \leq \lambda<n$, the space $\dot{\mathcal{N}}_{p, \lambda, q}^{s}\left(\mathbb{R}^{n}\right)$ is defined by

$$
\dot{\mathcal{N}}_{p, \lambda, q}^{s}\left(\mathbb{R}^{n}\right)=\left\{u \in \mathcal{Z}^{\prime}\left(\mathbb{R}^{n}\right) ; \quad\|u\|_{\dot{\mathcal{N}}_{p, \lambda, q}^{s}\left(\mathbb{R}^{n}\right)}<\infty\right\} .
$$

Here

$$
\|u\|_{\dot{\mathcal{N}}_{p, \lambda, q}^{s}\left(\mathbb{R}^{n}\right)}= \begin{cases}\left\{\sum_{j \in \mathbb{Z}} 2^{j \rho s}\left\|\dot{\Delta}_{j} u\right\|_{\mathrm{M}_{p}^{\lambda}}^{q}\right\}^{1 / q} & \text { for } q<\infty, \\ \sup _{j \in \mathbb{Z}} 2^{j s}\left\|\dot{\Delta}_{j} u\right\|_{\mathrm{M}_{p}^{\lambda}} & \text { for } q=\infty .\end{cases}
$$

The space $\mathcal{Z}^{\prime}\left(\mathbb{R}^{n}\right)$ denotes the topological dual of the space $\mathcal{Z}\left(\mathbb{R}^{n}\right)=\left\{f \in \mathcal{S}\left(\mathbb{R}^{n}\right) ; \partial^{\alpha} \widehat{f}(0)=\right.$ 0 for every multi-index $\alpha\}$, and can be identified to the quotient space $\mathcal{S}^{\prime}\left(\mathbb{R}^{n}\right) / \mathcal{P}$, where $\mathcal{P}$ represents the set of all polynomials on $\mathbb{R}^{n}$. We refer to [16, chap. 8] for more details.

Definition 3. (Homogeneous Fourier-Besov-Morrey spaces )

Let $s \in \mathbb{R}, 0 \leq \lambda<n, 1 \leq p<+\infty$ and $1 \leq q \leq+\infty$. The space $\mathcal{F} \dot{\mathcal{N}}_{p, \lambda, q}^{s}\left(\mathbb{R}^{n}\right)$ denotes the set of all $u \in \mathcal{Z}^{\prime}\left(\mathbb{R}^{n}\right)$ such that

$$
\|u\|_{\mathcal{F}_{p, \lambda, q}^{s}\left(\mathbb{R}^{n}\right)}=\left\{\sum_{j \in \mathbb{Z}} 2^{j q s}\left\|\widehat{\dot{\Delta}_{j} u}\right\|_{\mathrm{M}_{p}^{\lambda}}^{q}\right\}^{1 / q}<+\infty,
$$

with appropriate modifications made when $q=\infty$.

Note that the space $\mathcal{F} \dot{\mathcal{N}}_{p, \lambda, q}^{s}\left(\mathbb{R}^{n}\right)$ equipped with the norm (4) is a Banach space. Since $\mathrm{M}_{p}^{0}=L^{p}$, we have $\mathcal{F} \dot{\mathcal{N}}_{p, 0, q}^{s}=F \dot{B}_{p, q}^{s}, \mathcal{F} \dot{\mathcal{N}}_{1,0, q}^{s}=F \dot{B}_{1, q}^{s}=\dot{\mathcal{B}}_{q}^{s}$ and $\mathcal{F} \dot{\mathcal{N}}_{1,0,1}^{-1}=\chi^{-1}$ where $\dot{\mathcal{B}}_{q}^{s}$ is the Fourier-Herz space and $\chi^{-1}$ is the Lei-Lin space $[17,18]$.

Now, we give the definition of the mixed space-time spaces.

Definition 4. Let $s \in \mathbb{R}, 1 \leq p<\infty, 1 \leq q, \rho \leq \infty, 0 \leq \lambda<n$, and $I=[0, T), T \in(0, \infty]$. The space-time norm is defined on $u(t, x)$ by

$$
\|u(t, x)\|_{\mathcal{L}^{\rho}\left(I, \mathcal{F} \dot{\mathcal{N}}_{p, \lambda, q}^{s}\right)}=\left\{\sum_{j \in \mathbb{Z}} 2^{j q s}\left\|\widehat{\dot{\Delta}_{j} u}\right\|_{L^{\rho}\left(I, \mathrm{M}_{p}^{\lambda}\right)}^{q}\right\}^{1 / q},
$$

and denote by $\mathcal{L}^{\rho}\left(I, \mathcal{F} \dot{\mathcal{N}}_{p, \lambda, q, q}^{s}\right)$ the set of distributions in $S^{\prime}\left(\mathbb{R} \times \mathbb{R}^{n}\right) / \mathcal{P}$ with finite $\|\cdot\|_{\mathcal{L}^{\rho}\left(I, \mathcal{F} \dot{\mathcal{N}}_{p, \lambda, q}^{s}\right)}$ norm. According to Minkowski's inequality, we have

$$
\begin{array}{ll}
L^{\rho}\left(I ; \dot{\mathcal{N}}_{p, \lambda, q}^{s}\right) \hookrightarrow \mathcal{L}^{\rho}\left(I, \mathcal{F} \dot{\mathcal{N}}_{p, \lambda, q}^{s}\right), & \text { if } \rho \leq q, \\
\mathcal{L}^{\rho}\left(I, \mathcal{F} \dot{\mathcal{N}}_{p, \lambda, q}^{s}\right) \hookrightarrow L^{\rho}\left(I ; \mathcal{F} \dot{\mathcal{N}}_{p, \lambda, q}^{s}\right), & \text { if } \rho \geq q,
\end{array}
$$

where $\|u(t, x)\|_{L^{\rho}\left(I ; \mathcal{F} \dot{\mathcal{N}}_{p, \lambda, q}^{s}\right)}:=\left(\int_{I}\|u(\tau, \cdot)\|_{\mathcal{F N}_{p, \lambda, q}^{s}}^{\rho} d \tau\right)^{1 / \rho}$.

Our main result is the following theorem. 
Theorem 5. Let $\Omega \in \mathbb{R}, 0 \leq \lambda<3$ and $1 \leq q \leq 2$.

For $\max \left\{1, \frac{3-\lambda}{2}\right\} \leq p<\infty$ and $\frac{2}{3}<\alpha \leq \frac{2}{3}+\frac{1}{p^{\prime}}+\frac{\lambda}{3 p}$, there exists a positive time $T$ such that for any $u_{0} \in$ $\mathcal{F N}_{p, \lambda, q}^{1-2 \alpha+\frac{3}{p^{\prime}}+\frac{\lambda}{p}}$ and $\nabla \cdot u_{0}=0$, the Equation (1) admits a unique local solution $u$ in $\mathcal{L}^{4}\left([0, T), \mathcal{F} \dot{\mathcal{N}}_{p, \lambda, q}^{1-\frac{3}{2} \alpha+\frac{3}{p^{\prime}}+\frac{\lambda}{p}}\right)$.

Furthermore, for all $1 \leq p<\infty$ and $\frac{1}{2}<\alpha \leq 1+\frac{3}{2 p^{\prime}}+\frac{\lambda}{2 p}$ there exists a constant $C_{0}(p, q)$ such that for any $u_{0} \in \mathcal{F N}_{p, \lambda, q}^{1-2 \alpha+\frac{3}{p^{\prime}}+\frac{\lambda}{p}}$ satisfying $\nabla \cdot u_{0}=0$ and $\left\|u_{0}\right\|_{\mathcal{F N}_{p, \lambda, q}^{1-2 \alpha+\frac{3}{p^{\prime}}+\frac{\lambda}{p}}}<C_{0} \mu$, the Equation (1) admits a unique global solution

$$
u \in \mathcal{C}\left([0, \infty) ; \dot{F}^{1-2 \alpha+\frac{3}{p^{\prime}}+\frac{\lambda}{p}}\right) \cap \mathcal{L}^{1}\left([0, \infty), \mathcal{F}^{1+\lambda, q} \dot{\mathcal{N}}_{p, \lambda, q}^{1+\frac{3}{p^{\prime}}+\frac{\lambda}{p}}\right)
$$

and it satisfies

$$
\|u\|_{\mathcal{L}^{\infty}\left([0, \infty) ; \mathcal{F} \dot{\mathcal{N}}_{p, \lambda, q}^{1-2 \alpha+\frac{3}{p^{\prime}}+\frac{\lambda}{p}}\right)}+\mu\|u\| \mathcal{L}_{\mathcal{L}^{1}\left([0, \infty), \mathcal{F} \dot{\mathcal{N}}_{p, \lambda, q}^{1+\frac{3}{p^{\prime}}+\frac{\lambda}{p}}\right)} \leq 2 C\left\|u_{0}\right\|_{\mathcal{F N}_{p, \lambda, q}^{1-2 \alpha+\frac{3}{p^{\prime}}+\frac{\lambda}{p}}}
$$

where $C$ is a positive constant.

Now, we give some remarks about this result.

Remark 1. When $\alpha=1$, there are a different results which investigate the existence of a unique global solution to the Navier-stokes equations with Coriolis forces, especially in Fourier-Herz spaces $\dot{\mathcal{B}}_{2}^{-1}[6]$, in Lei-lin spaces $\chi^{-1}[19,20]$ and in Fourier-Besov spaces $\mathrm{FB}_{p, \infty}^{2-\frac{3}{p}}$ [13]. Theorem 5 is an extension and an improvement of these works to the Fourier-Besov-Morrey space $\mathcal{F} \mathcal{N}_{p, \lambda, q}^{1-2 \alpha+\frac{3}{p^{\prime}}+\frac{\lambda}{p}}\left(\mathbb{R}^{3}\right)$.

We note that the fractional Navier-Stokes-Coriolis system is well-posed uniformly in the sense that the smallness condition is independent of $\Omega$.

Theorem 6. Let $\Omega \in \mathbb{R}, 1 \leq p, q \leq 2,0 \leq \lambda \leq 3-\frac{3}{2} p$ and $\frac{5}{6}<\alpha \leq 1$. Assume that $u \in \mathcal{C}\left([0, \infty) ; \mathcal{F} \dot{\mathcal{N}}_{p, \lambda, q}^{1-2 \alpha+\frac{3}{p^{\prime}}+\frac{\lambda}{p}}\right)$ is a global solution of the system (1) given by Theorem 5 , then

$$
\lim _{t \rightarrow \infty} \sup \|u(t)\| \underset{\mathcal{F N}_{p, \lambda, q}^{1-2 \alpha+\frac{3}{p^{\prime}}+\frac{\lambda}{p}}}{ }=0 .
$$

Remark 2. Theorem 6 specifies the asymptotic behavior of a given global solution for (1) in the space $\mathcal{F N}_{p, \lambda, q}^{1-2 \alpha+\frac{3}{p^{\prime}}+\frac{\lambda}{p}}$. The long time decay property is also valid in the case $\Omega=0$ where the Equation $(1)$ is reduced to the fractional Navier-Stokes equation. At present, we are unable to establish that (6) still holds true for $\frac{1}{2}<\alpha \leq \frac{5}{6}$. The principal reason is that the proof is largely based on the lemma 14 .

Theorem 7. Let $T^{*}$ denote the maximal time of existence of a solution $u$ in

$$
\begin{gathered}
\mathcal{L}^{\infty}\left(\left[0, T^{*}\right) ; \mathcal{F N}_{p, \lambda, q}^{1-2 \alpha+\frac{3}{p^{\prime}}+\frac{\lambda}{p}}\right) \cap \mathcal{L}^{1}\left(\left[0, T^{*}\right), \mathcal{F N}_{p, \lambda, q}^{1+\frac{3}{p^{\prime}}+\frac{\lambda}{p}}\right) \text {. If } T^{*}<\infty \text {, then } \\
\|u\|_{\mathcal{L}^{1}\left(\left[0, T^{*}\right), \mathcal{F} \dot{\mathcal{N}}_{p, \lambda, q}^{1+\frac{3}{p^{\prime}}+\frac{\lambda}{p}}\right)}=\infty .
\end{gathered}
$$

Besides; if $u \in C\left(\mathbb{R}^{+}, \mathcal{F} \dot{\mathcal{N}}_{p, \lambda, q}^{1-2 \alpha+\frac{3}{p^{\prime}}+\frac{\lambda}{p}}\right)$ is a global solution of (1), and for all $v_{0} \in \mathcal{F} \dot{\mathcal{N}}_{p, \lambda, q}^{1-2 \alpha+\frac{3}{p^{\prime}}+\frac{\lambda}{p}}$ satisfying

$$
\left\|v_{0}-u_{0}\right\|_{\mathcal{F \mathcal { N }}_{p, \lambda, q}^{1-2 \alpha+\frac{3}{p^{\prime}}}+\frac{\lambda}{p}}<C_{0} \frac{\mu}{8} \exp \left\{-\int_{0}^{\infty} \frac{1}{\mathcal{C}_{0}}\left(|\Omega|+\|u\|_{\mathcal{F \mathcal { N }}_{p, \lambda, q}^{1+\frac{3}{p^{\prime}}}}\left(\frac{\lambda}{p}\right)\right\}\right.
$$


for some constant $C_{0}$ sufficiently small, then the fractional Navier-Stokes-Coriolis system starting by $v_{0}$ has a global solution $v$ fulfilling the inequality

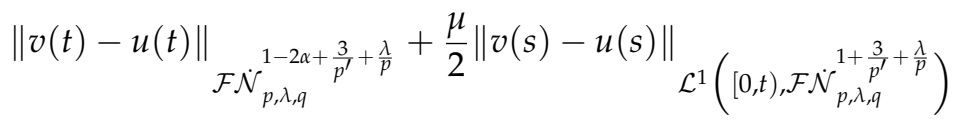

$$
\begin{aligned}
& <C\left\|v_{0}-u_{0}\right\|_{\mathcal{F N}_{p, \lambda, q}^{1-2 \alpha+\frac{3}{p^{\prime}}}+\frac{\lambda}{p}} \exp \left\{\int_{0}^{\infty} C\left(|\Omega|+\|u\|_{\mathcal{F N}_{p, \lambda, \lambda}^{1+\frac{3}{p^{\prime}}}+\frac{\lambda}{p}}\right)\right\}
\end{aligned}
$$

where $C$ is a positive constant.

Remark 3. In the case $\Omega=0$ and $\alpha=1$, the result of stability of global solutions for the usual Navier-Stokes equations is developed by several researchers in different function spaces such as $\mathrm{H}^{1}, \mathrm{~L}^{3}, \dot{\mathrm{B}}_{p, q}^{\frac{3}{p}-1}$ and $\chi^{-1}$ [11,21-23]. To show Theorem 7, we adapt the method of the above works to our problem (1) in the spaces $\mathcal{F} \dot{\mathcal{N}}_{p, \lambda, q}^{1-2 \alpha+\frac{3}{p^{\prime}}+\frac{\lambda}{p}}$. Theorem 7 extends the works of $[11,21,23]$ to a more general frame.

We finish this section with a Bernstein type lemma in Fourier variables in Morrey spaces.

Lemma 8 ([24]). Let $1 \leq q \leq p<\infty, 0 \leq \lambda_{1}, \lambda_{2}<n, \frac{n-\lambda_{1}}{p} \leq \frac{n-\lambda_{2}}{q}$, and let $\gamma$ be a multiindex. If supp $(\widehat{f}) \subset$ $\left\{|\xi| \leq A 2^{j}\right\}$ then there is a constant $C>0$ independent of $f$ and $j$ such that

$$
\left\|(i \xi)^{\gamma} \widehat{f}\right\|_{\mathrm{M}_{q}^{\lambda_{2}}} \leq C 2^{j|\gamma|+j\left(\frac{n-\lambda_{2}}{q}-\frac{n-\lambda_{1}}{p}\right)}\|\widehat{f}\|_{\mathrm{M}_{p}^{\lambda_{1}}}
$$

\section{Well-posedness}

In order to solve (1), we consider the following integral equation:

$$
u(t)=T_{\Omega, \alpha}(t) u_{0}-\int_{0}^{t} T_{\Omega, \alpha}(t-\tau) \mathbb{P} \nabla \cdot(u \otimes u) d \tau,
$$

where $\mathbb{P}=\left(\delta_{i j}+R_{i} R_{j}\right)_{1 \leq i, j \leq 3}$ denotes the Helmholtz projection onto the divergence-free vector fields, which is a pseudo differential operator of order 0 , and $T_{\Omega, \alpha}(\cdot)$ denotes the Stokes-Coriolis semigroup corresponding to the following linear Stokes problem with Coriolis force

$$
\left\{\begin{array}{l}
u_{t}+\mu(-\Delta)^{\alpha} u+\Omega e_{3} \times u+\nabla \pi=0 \quad(t, x) \in \mathbb{R}^{+} \times \mathbb{R}^{3}, \\
\nabla . u=0 \\
u(0, x)=u_{0}(x) \quad x \in \mathbb{R}^{3} .
\end{array}\right.
$$

Besides, $T_{\Omega, 1}(\cdot)$ is given explicitly by

$$
T_{\Omega, 1}(t) f=\mathcal{F}^{-1}\left[\cos \left(\Omega \frac{\xi_{3}}{|\xi|} t\right) I+\sin \left(\Omega \frac{\xi_{3}}{|\xi|} t\right) R(\xi)\right] *\left(e^{\mu \Delta t} f\right)
$$

for $t \geq 0$ and divergence-free vector fields $f$. Here, $I$ is the identity matrix in $\mathbb{R}^{3}$ and $R(\xi)$ is the skew-symmetric matrix symbol related to the Riesz transform, which is defined by

$$
R(\xi):=\frac{1}{|\xi|}\left(\begin{array}{lcr}
0 & \xi_{3} & -\xi_{2} \\
-\xi_{3} & 0 & \xi_{1} \\
\xi_{2} & -\xi_{1} & 0
\end{array}\right) .
$$

We refer to Babin-Mahalov-Nikolaenko [25-27], Giga-Inui-Mahalov-Saal [8] and Hieber- Shibata [3] for the derivation of the explicit form of $T_{\Omega, 1}(\cdot)$. 
For $\alpha \in\left(\frac{2}{3}, \frac{2}{3}+\frac{1}{p^{\prime}}+\frac{\lambda}{3 p^{\prime}}\right]$, the same argument given in $[3,10]$ gives

$$
T_{\Omega, \alpha}(t) f=\mathcal{F}^{-1}\left[\cos \left(\Omega \frac{\xi_{3}}{|\xi|} t\right) I+\sin \left(\Omega \frac{\xi_{3}}{|\xi|} t\right) R(\xi)\right] *\left(e^{-\mu(-\Delta)^{\alpha} t} f\right) .
$$

The first estimate corresponds to the Stokes-Coriolis semigroup $T_{\Omega, \alpha}$.

Lemma 9. Let $0<T \leq \infty, s \in \mathbb{R}, 0 \leq \lambda<3,1 \leq p<\infty, 1 \leq q, \rho, r \leq \infty$ and $f \in \mathcal{L}^{r}\left([0, T), \mathcal{F} \dot{\mathcal{N}}_{p, \lambda, q}^{s}\right)$. There exists a constant $C>0$ such that

$$
\left\|\int_{0}^{t} T_{\Omega, \alpha}(t-\tau) f(\tau) d \tau\right\|_{\mathcal{L}^{\rho}\left([0, T), \mathcal{F} \dot{\mathcal{N}}_{p, \lambda, q}^{s}\right)} \leq C\|f\|_{\mathcal{L}^{r}\left([0, T), \mathcal{F} \dot{\mathcal{N}}_{p, \lambda, q}^{s-2 \alpha-\frac{2 \alpha}{\rho}+\frac{2 \alpha}{r}}\right)} .
$$

Proof. Set $1+\frac{1}{\rho}=\frac{1}{\tilde{\rho}}+\frac{1}{r}$. The definition of the space-time norm of $\mathcal{L}^{\rho}\left([0, T), \mathcal{F \mathcal { N }}_{p, \lambda, q}^{s}\right)$ and Young's inequality give

$$
\begin{aligned}
\left\|\int_{0}^{t} T_{\Omega, \alpha}(t-\tau) f(\tau) d \tau\right\|_{\mathcal{L}^{\rho}\left([0, T), \mathcal{F} \dot{\mathcal{N}}_{p, \lambda, q}^{s}\right)} & =\left\{\sum_{j \in \mathbb{Z}} 2^{j q s}\left(\int_{0}^{T}\left\|\varphi_{j} \int_{0}^{t} \mathcal{F}\left(T_{\Omega, \alpha}(t-\tau) f\right)(\tau) d \tau\right\|_{\mathrm{M}_{p}^{\lambda}}^{\rho} d t\right)^{\frac{q}{\rho}}\right\}^{1 / q} \\
& \leq\left\{\sum_{j \in \mathbb{Z}} 2^{j q s}\left(\int_{0}^{T}\left\|\varphi_{j} \int_{0}^{t} e^{-\mu|\xi|^{2 \alpha}(t-\tau)} \hat{f}(\tau) d \tau\right\|_{\mathrm{M}_{p}^{\lambda}}^{\rho} d t\right)^{\frac{q}{\rho}}\right\}^{1 / q} \\
& \leq\left\{\sum_{j \in \mathbb{Z}} 2^{j q s}\left(\int_{0}^{T}\left\|\varphi_{j} \int_{0}^{t} e^{-\mu 2^{2 \alpha j}(t-\tau)} \hat{f}(\tau) d \tau\right\|_{\mathrm{M}_{p}^{\lambda}}^{\rho} d t\right)^{\frac{q}{\rho}}\right\}^{1 / q} \\
& \leq\left\{\sum_{j \in \mathbb{Z}} 2^{j q s}\left(\int_{0}^{T} e^{-t \mu \tilde{\rho} 2^{2 \alpha j}} d t\right)^{\frac{q}{\rho}}\left\|\varphi_{j} \hat{f}(\tau)\right\|_{L^{r}\left([0, T), \mathrm{M}_{p}^{\lambda}\right)}^{q}\right\}^{1 / q} \\
& \leq C\left\{\sum_{j \in \mathbb{Z}} 2^{j q\left(s-2 \alpha-\frac{2 \alpha}{\rho}+\frac{2 \alpha}{r}\right)}\left\|\varphi_{j} \hat{f}(\tau)\right\|_{L^{r}\left([0, T), \mathrm{M}_{p}^{\lambda}\right)}^{q}\right\}^{1 / q} \\
& \leq C\|f\|_{\mathcal{L}^{r}\left([0, T), \mathcal{F} \dot{\mathcal{N}}_{p, \lambda, q}^{s-2 \alpha}\right.}
\end{aligned}
$$

Lemma 10. Let $I=[0, T), 0<T \leq \infty, 0 \leq \lambda<3,1 \leq p<\infty, 1 \leq q \leq \infty$ and $u_{0} \in \mathcal{F} \mathcal{N}_{p, \lambda, q}^{1-2 \alpha+\frac{3}{p^{\prime}}+\frac{\lambda}{p}}\left(\mathbb{R}^{3}\right)$. Then there exists a constant $C>0$ such that

$$
\begin{aligned}
& \left\|T_{\Omega, \alpha}(t) u_{0}\right\|_{\mathcal{L}^{\infty}\left([0, T), \mathcal{F} \dot{\mathcal{N}}_{p, \lambda, q}^{1-2 \alpha+\frac{3}{p^{\prime}}+\frac{\lambda}{p}}\right)} \leq C\left\|u_{0}\right\|_{\mathcal{F} \dot{\mathcal{N}}_{p, \lambda, q}^{1-2 \alpha+\frac{3}{p^{\prime}}}+\frac{\lambda}{p}{ }^{\prime}} \\
& \left\|T_{\Omega, \alpha}(t) u_{0}\right\|_{\mathcal{L}^{1}\left([0, T), \mathcal{F} \dot{\mathcal{N}}_{p, \lambda, q}^{1+\frac{3}{p^{\prime}}+\frac{\lambda}{p}}\right)} \leq C\left\|u_{0}\right\|_{\mathcal{F}_{\dot{\mathcal{N}}_{p, \lambda, q}}^{1-2 \alpha+\frac{3}{p^{\prime}}+\frac{\lambda}{p}}} \\
& \left\|T_{\Omega, \alpha}(t) u_{0}\right\|_{\mathcal{L}^{4}\left([0, T), \mathcal{F} \dot{\mathcal{N}}_{p, \lambda, q}^{1-\frac{3}{2} \alpha+\frac{3}{p^{\prime}}+\frac{\lambda}{p}}\right)} \leq C\left\|u_{0}\right\|_{\mathcal{F N}_{\dot{N}_{p, \lambda, q}}{ }^{1-2 \alpha+\frac{3}{p^{\prime}}+\frac{\lambda}{p}} .} .
\end{aligned}
$$

Proof. To prove the first inequality (9), it suffices to write that

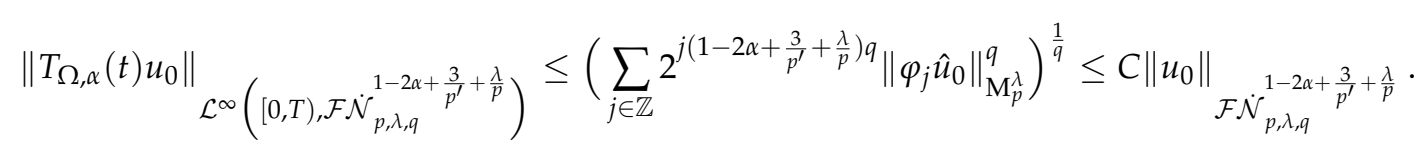

In order to prove the second estimate (10), we write

$$
\left\|T_{\Omega, \alpha}(t) u_{0}\right\|_{\mathcal{L}^{1}\left([0, T), \mathcal{F} \dot{\mathcal{N}}_{p, \lambda, q}^{1+\frac{3}{p^{2}}+\frac{\lambda}{p}}\right)} \leq\left(\sum_{j \in \mathbb{Z}} 2^{j\left(1+\frac{3}{p^{\prime}}+\frac{\lambda}{p}\right) q}\left(\int_{0}^{T} e^{-t \mu 2^{2 \alpha j}}\left\|\varphi_{j} \hat{u}_{0}\right\|_{\mathrm{M}_{p}^{\lambda}} d t\right)^{q}\right)^{\frac{1}{q}} \leq C\left\|u_{0}\right\|_{\mathcal{\mathcal { N }} \dot{\mathcal{N}}_{p, \lambda, q}^{1-2 \alpha+\frac{3}{p^{\prime}}}+\frac{\lambda}{p}}
$$


To estimate the inequality (11), it suffices to write that

$$
\begin{aligned}
\left\|T_{\Omega, \alpha}(t) u_{0}\right\|_{\mathcal{L}^{4}\left([0, T), \mathcal{F} \dot{\mathcal{N}}_{p, \lambda, q}^{1-\frac{3}{2} \alpha+\frac{3}{p^{\prime}}+\frac{\lambda}{p}}\right)} & \leq\left(\sum_{j \in \mathbb{Z}} 2^{j\left(1-\frac{3}{2} \alpha+\frac{3}{p^{\prime}}+\frac{\lambda}{p}\right) q}\left(\int_{0}^{T} e^{-t \mu 2^{2 \alpha j+2}}\left\|\varphi_{j} \hat{u}_{0}\right\|_{\mathrm{M}_{p}^{\lambda}}^{4} d t\right)^{\frac{q}{4}}\right)^{\frac{1}{q}} \\
& \leq C\left(\sum_{j \in \mathbb{Z}} 2^{j\left(1-\frac{3}{2} \alpha+\frac{3}{p^{\prime}}+\frac{\lambda}{p}\right) q} 2^{-\frac{1}{2} \alpha j q}\left\|\varphi_{j} \hat{u}_{0}\right\|_{\mathrm{M}_{p}^{\lambda}}^{q}\right)^{\frac{1}{q}} \leq C\left\|u_{0}\right\|_{\mathcal{F} \dot{\mathcal{N}}_{p, \lambda, q}^{1-2 \alpha+\frac{3}{p^{\prime}}+\frac{\lambda}{p}}} .
\end{aligned}
$$

Proposition 11. Let $0 \leq \lambda<3, \max \left\{1, \frac{3-\lambda}{2}\right\} \leq p<\infty, 1 \leq q \leq 2, I=[0, T), 0<T \leq+\infty$ and $\frac{2}{3}<\alpha \leq$ $\frac{2}{3}+\frac{1}{p^{\prime}}+\frac{\lambda}{3 p}$, and set

$$
Y=\mathcal{L}^{4}\left(I, \mathcal{F} \dot{\mathcal{N}}_{p, \lambda, q}^{1-\frac{3}{2} \alpha+\frac{3}{p^{\prime}}+\frac{\lambda}{p}}\right)
$$

there exists a constant $C=C(p, q)>0$ depending on $p, q$ such that

$$
\|u v\|_{\mathcal{L}^{2}\left(I, \mathcal{F} \dot{\mathcal{N}}_{p, \lambda, q}^{2-3 \alpha+\frac{3}{p^{\prime}}+\frac{\lambda}{p}}\right)} \leq C\|u\|_{Y}\|v\|_{Y}
$$

Proof. We need to introduce some notations about the standard localization operators. We set

$$
u_{j}=\dot{\Delta}_{j} u, \quad \dot{S}_{j} u=\sum_{k \leq j-1} \dot{\Delta}_{k} u, \quad \tilde{\dot{\Delta}}_{j} u=\sum_{|k-j| \leq 1} \dot{\Delta}_{k} u, \quad j \in \mathbb{Z} .
$$

Applying Bony paraproduct decomposition and quasi-orthogonality property for Littlewood-Paley decomposition, for fixed $j$, we obtain

$$
\begin{aligned}
\dot{\Delta}_{j}(u v) & =\sum_{|k-j| \leq 4} \dot{\Delta}_{j}\left(\dot{S}_{k-1} u \dot{\Delta}_{k} v\right)+\sum_{|k-j| \leq 4} \dot{\Delta}_{j}\left(\dot{S}_{k-1} v \dot{\Delta}_{k} u\right)+\sum_{k \geq j-3} \dot{\Delta}_{j}\left(\dot{\Delta}_{k} u \widetilde{\dot{\Delta}}_{k} v\right) \\
& =I_{j}+I I_{j}+I I I_{j} .
\end{aligned}
$$

The triangular inequality gives

$$
\begin{aligned}
\|u v\|_{\mathcal{L}^{2}\left(I, \mathcal{F} \dot{N}_{p, \lambda, q}^{\left.2-3 \alpha+\frac{3}{p^{\prime}}+\frac{\lambda}{p}\right)} \lesssim\right.} & \left\{\sum_{j \in \mathbb{Z}} 2^{j\left(2-3 \alpha+\frac{3}{p^{\prime}}+\frac{\lambda}{p}\right) q}\left\|\widehat{I}_{j}\right\|_{L^{2}\left(I, \mathrm{M}_{p}^{\lambda}\right)}^{q}\right\}^{1 / q}+\left\{\sum_{j \in \mathbb{Z}} 2^{j\left(2-3 \alpha+\frac{3}{p^{\prime}}+\frac{\lambda}{p}\right) q}\left\|\widehat{I I}_{j}\right\|_{L^{2}\left(I, \mathrm{M}_{p}^{\lambda}\right)}^{q}\right\}^{1 / q} \\
& +\left\{\sum_{j \in \mathbb{Z}} 2^{j\left(2-3 \alpha+\frac{3}{p^{\prime}}+\frac{\lambda}{p}\right) q}\|\widehat{I I I}\|_{L^{2}\left(I, \mathrm{M}_{p}^{\lambda}\right)}^{q}\right\}^{1 / q}
\end{aligned}
$$

We evaluate the above three terms individually. First, using Young's inequality (3) and Lemma 8 with $|\gamma|=0$, we get

$$
\begin{aligned}
& 2^{j\left(2-3 \alpha+\frac{3}{p^{\prime}}+\frac{\lambda}{p}\right)}\left\|\widehat{I}_{j}\right\|_{L^{2}\left(I, \mathrm{M}_{p}^{\lambda}\right)} \leq 2^{j\left(2-3 \alpha+\frac{3}{p^{\prime}}+\frac{\lambda}{p}\right)} \sum_{|k-j| \leq 4}\left\|\dot{S}_{k-1} u \dot{\Delta}_{k} v\right\|_{L^{2}\left(I, \mathrm{M}_{p}^{\lambda}\right)} \\
& \leq 2^{j\left(2-3 \alpha+\frac{3}{p^{\prime}}+\frac{\lambda}{p}\right)} \sum_{|k-j| \leq 4}\left\|\widehat{v}_{k}\right\|_{L^{4}\left(I, \mathrm{M}_{p}^{\lambda}\right)} \sum_{l \leq k-2}\left\|\widehat{u}_{l}\right\|_{L^{4}\left(I, L^{1}\right)} \\
& \leq 2^{j\left(2-3 \alpha+\frac{3}{p^{\prime}}+\frac{\lambda}{p}\right)} \sum_{|k-j| \leq 4}\left\|\widehat{v}_{k}\right\|_{L^{4}\left(I, \mathrm{M}_{p}^{\lambda}\right)} \sum_{l \leq k-2} 2^{l\left(\frac{3}{p^{\prime}}+\frac{\lambda}{p}\right)}\left\|\widehat{u}_{l}\right\|_{L^{4}\left(I, \mathrm{M}_{p}^{\lambda}\right)} \\
& \leq 2^{j\left(2-3 \alpha+\frac{3}{p^{\prime}}+\frac{\lambda}{p}\right)} \sum_{|k-j| \leq 4}\left\|\widehat{v}_{k}\right\|_{L^{4}\left(I, \mathrm{M}_{p}^{\lambda}\right)} \sum_{l \leq k-2} 2^{l\left(\frac{3}{p^{\prime}}+\frac{\lambda}{p}\right)} 2^{\left(1-\frac{3}{2} \alpha\right) l} 2^{\left(\frac{3}{2} \alpha-1\right) l}\left\|\widehat{u}_{l}\right\|_{L^{4}\left(I, \mathrm{M}_{p}^{\lambda}\right)} \\
& \leq 2^{j\left(2-3 \alpha+\frac{3}{p^{\prime}}+\frac{\lambda}{p}\right)} \sum_{|k-j| \leq 4}\left\|\widehat{v}_{k}\right\|_{L^{4}\left(I, \mathrm{M}_{p}^{\lambda}\right)}\left(\sum_{l \leq k-2} 2^{\left(\frac{3}{2} \alpha-1\right) l q^{\prime}}\right)^{\frac{1}{q^{\prime}}}\|u\|_{\mathcal{L}^{4}\left(I, \mathcal{F} \dot{\mathcal{N}}_{p, \lambda, q}^{1-\frac{3}{2} \alpha+\frac{3}{p^{\prime}}+\frac{\lambda}{p}}\right)}
\end{aligned}
$$




$$
\lesssim 2^{j\left(2-3 \alpha+\frac{3}{p^{\prime}}+\frac{\lambda}{p}\right)} \sum_{|k-j| \leq 4} 2^{\left(\frac{3}{2} \alpha-1\right) k}\left\|\widehat{v}_{k}\right\|_{L^{4}\left(I, \mathrm{M}_{p}^{\lambda}\right)}\|u\|_{\mathcal{L}^{4}\left(I, \mathcal{F} \dot{\mathcal{N}}_{p, \lambda, q}^{1-\frac{3}{2} \alpha+\frac{3}{p^{\prime}}+\frac{\lambda}{p}}\right)}
$$

Applying $l^{q}$-norm on both sides in the above estimate we obtain

$$
\begin{aligned}
\left\{\sum_{j \in \mathbb{Z}} 2^{j\left(2-3 \alpha+\frac{3}{p^{\prime}}+\frac{\lambda}{p}\right) q}\left\|\widehat{I}_{j}\right\|_{L^{2}\left(I, \mathrm{M}_{p}^{\lambda}\right)}^{q}\right\}^{1 / q} & \lesssim\left\{\sum_{j \in \mathbb{Z}}\left(\sum_{|k-j| \leq 4} 2^{k\left(1-\frac{3}{2} \alpha+\frac{3}{p^{\prime}}+\frac{\lambda}{p}\right)} 2^{(j-k)\left(2-3 \alpha+\frac{3}{p^{\prime}}+\frac{\lambda}{p}\right)}\left\|\widehat{v}_{k}\right\|_{L^{4}\left(I, \mathrm{M}_{p}^{\lambda}\right)}\right)^{q}\right\}^{1 / q} \\
& \times\|u\|_{\mathcal{L}^{4}\left(I, \mathcal{F} \dot{\mathcal{N}}_{p, \lambda, q}^{1-\frac{3}{2} \alpha+\frac{3}{p^{\prime}}+\frac{\lambda}{p}}\right)} \lesssim\|u\|_{\mathcal{L}^{4}\left(I, \mathcal{F} \dot{\mathcal{N}}_{p, \lambda, q}^{1-\frac{3}{2} \alpha+\frac{3}{p^{\prime}}+\frac{\lambda}{p}}\right)}\|v\|_{\mathcal{L}^{4}\left(I, \mathcal{F} \dot{\mathcal{N}}_{p, \lambda, q}^{1-\frac{3}{2} \alpha+\frac{3}{p^{\prime}}+\frac{\lambda}{p}}\right)}
\end{aligned}
$$

Likewise, we prove that

$$
\begin{aligned}
\left\{\sum_{j \in \mathbb{Z}} 2^{j\left(2-3 \alpha+\frac{3}{p^{\prime}}+\frac{\lambda}{p}\right) q}\left\|\widehat{I I}_{j}\right\|_{L^{2}\left(I, \mathrm{M}_{p}^{\lambda}\right)}^{q}\right\}^{1 / q} & \lesssim\left\{\sum_{j \in \mathbb{Z}}\left(\sum_{|k-j| \leq 4} 2^{k\left(1-\frac{3}{2} \alpha+\frac{3}{p^{\prime}}+\frac{\lambda}{p}\right)} 2^{(j-k)\left(2-3 \alpha+\frac{3}{p^{\prime}}+\frac{\lambda}{p}\right)}\left\|\widehat{u}_{k}\right\|_{L^{4}\left(I, \mathrm{M}_{p}^{\lambda}\right)}\right)^{q}\right\}^{1 / q} \\
& \times \quad\|v\|_{\mathcal{L}^{4}\left(I, \mathcal{F} \dot{\mathcal{N}}_{p, \lambda, q}^{1-\frac{3}{2} \alpha+\frac{3}{p^{\prime}}+\frac{\lambda}{p}}\right)} \lesssim\|v\|_{\mathcal{L}^{4}\left(I, \mathcal{F} \dot{\mathcal{N}}_{p, \lambda, q}^{1-\frac{3}{2} \alpha+\frac{3}{p^{\prime}}+\frac{\lambda}{p}}\right)}\|u\| \mathcal{L}_{\mathcal{L}^{4}\left(I, \mathcal{F} \dot{\mathcal{N}}_{p, \lambda, q}^{1-\frac{3}{2} \alpha+\frac{3}{p^{\prime}}+\frac{\lambda}{p}}\right)} .
\end{aligned}
$$

To estimate $I I I_{j}$, the so-called "remainder term", we use a different approach. Let

$$
I I I_{j k}:=\dot{\Delta}_{j}\left(\sum_{|i-k| \leq 1} \dot{\Delta}_{i} v \dot{\Delta}_{k} u\right)=\sum_{i=-1}^{1} \dot{\Delta}_{j}\left(\dot{\Delta}_{k} u \dot{\Delta}_{i+k} v\right) .
$$

First, we use Young's inequality (3) and Lemma 8 with $|\gamma|=0$ to obtain

$$
\begin{aligned}
& 2^{j\left(2-3 \alpha+\frac{3}{p^{\prime}}+\frac{\lambda}{p}\right)}\left\|\widehat{I I I_{j}}\right\|_{L^{2}\left(I, \mathrm{M}_{p}^{\lambda}\right)} \leq 2^{j\left(2-3 \alpha+\frac{3}{p^{\prime}}+\frac{\lambda}{p}\right)} \sum_{k \geq j-3}\left\|\widehat{I I I_{j k}}\right\|_{L^{2}\left(I, \mathrm{M}_{p}^{\lambda}\right)} \\
& \leq 2^{j\left(2-3 \alpha+\frac{3}{p^{\prime}}+\frac{\lambda}{p}\right)} \sum_{k \geq j-3|k-i| \leq 1}\left\|\widehat{\Delta_{k} u \dot{\Delta}_{i} v}\right\|_{L^{2}\left(I, \mathrm{M}_{p}^{\lambda}\right)} \\
& \leq 2^{j\left(2-3 \alpha+\frac{3}{p^{\prime}}+\frac{\lambda}{p}\right)} \sum_{k \geq j-3} \sum_{|k-i| \leq 1}\left\|\widehat{u}_{k}\right\|_{L^{4}\left(I, \mathrm{M}_{p}^{\lambda}\right)}\left\|\widehat{v}_{i}\right\|_{L^{4}\left(I, L^{1}\right)} \\
& \leq 2^{j\left(2-3 \alpha+\frac{3}{p^{\prime}}+\frac{\lambda}{p}\right)} \sum_{k \geq j-3} \sum_{|k-i| \leq 1} 2^{i\left(\frac{3}{p^{\prime}}+\frac{\lambda}{p}\right)}\left\|\widehat{u}_{k}\right\|_{L^{4}\left(I, \mathrm{M}_{p}^{\lambda}\right)}\left\|\widehat{v}_{i}\right\|_{L^{4}\left(I, \mathrm{M}_{p}^{\lambda}\right)} \\
& \leq C 2^{j\left(2-3 \alpha+\frac{3}{p^{\prime}}+\frac{\lambda}{p}\right)} \sum_{k \geq j-3}\left(\sum_{|k-i| \leq 1} 2^{i q\left(1-\frac{3}{2} \alpha+\frac{3}{p^{\prime}}+\frac{\lambda}{p}\right)}\left\|\widehat{v}_{i}\right\|_{L^{4}\left(I, \mathrm{M}_{p}^{\lambda}\right)}^{q}\right)^{\frac{1}{q}} \\
& \times 2^{\left(\frac{3}{2} \alpha-1\right) k}\left\|\widehat{u}_{k}\right\|_{L^{4}\left(I, \mathrm{M}_{p}^{\lambda}\right)} \\
& \leq C\|v\|_{\mathcal{L}^{4}\left(I, \mathcal{F} \dot{\mathcal{N}}_{p, \lambda, q}^{1-\frac{3}{2} \alpha+\frac{3}{p^{\prime}}+\frac{\lambda}{p}}\right)} \sum_{k \geq j-3} 2^{(j-k)\left(2-3 \alpha+\frac{3}{p^{\prime}}+\frac{\lambda}{p}\right)} 2^{k\left(1-\frac{3}{2} \alpha+\frac{3}{p^{\prime}}+\frac{\lambda}{p}\right)}\left\|\widehat{u}_{k}\right\|_{L^{4}\left(I, \mathrm{M}_{p}^{\lambda}\right)}
\end{aligned}
$$

When $2-3 \alpha+\frac{3}{p^{\prime}}+\frac{\lambda}{p}>0$, we take the $l^{q}$-norm on both sides in the above estimate, and then we apply Young's inequality for series to get

$$
\left(\sum_{j \in \mathbb{Z}} 2^{j\left(2-3 \alpha+\frac{3}{p^{\prime}}+\frac{\lambda}{p}\right) q}\|\widehat{I I I}\|_{L^{2}\left(I, \mathrm{M}_{p}^{\lambda}\right)}^{q}\right)^{\frac{1}{q}} \leq C\|v\|_{\mathcal{L}^{4}\left(I, \mathcal{F} \dot{\mathcal{N}}_{p, \lambda, q}^{1-\frac{3}{2} \alpha+\frac{3}{p^{\prime}}+\frac{\lambda}{p}}\right)}\|u\| \mathcal{L}_{\mathcal{L}^{4}\left(I, \mathcal{F} \dot{\mathcal{N}}_{p, \lambda, q}^{1-\frac{3}{2} \alpha+\frac{3}{p^{\prime}}+\frac{\lambda}{p}}\right)} .
$$

For the case $2-3 \alpha+\frac{3}{p^{\prime}}+\frac{\lambda}{p}=0$, we write 


$$
\begin{aligned}
& \left(\sum_{j \in \mathbb{Z}}\left\|\widehat{I I I}_{j}\right\|_{L^{2}\left(I, \mathrm{M}_{p}^{\lambda}\right)}^{q}\right)^{\frac{1}{q}} \leq \sum_{j \in \mathbb{Z}}\left(\sum_{k \geq j-3}\left(\int_{I}\left\|\varphi_{j}(\xi) \times \sum_{i=-1}^{1} \hat{u}_{k} * \hat{v}_{k+i}\right\|_{\mathrm{M}_{p}^{\lambda}}^{2}\right)^{\frac{1}{2}}\right) \\
& \left.\leq \sup _{\xi}\left(\sum_{j \in \mathbb{Z}} \varphi_{j}(\xi)\right) \sum_{k \in \mathbb{Z}}\left(\int_{I}\left\|\sum_{i=-1}^{1} \hat{u}_{k} * \hat{v}_{k+i}\right\|_{\mathrm{M}_{p}^{\lambda}}^{2}\right)^{\frac{1}{2}}\right) \\
& \leq \sum_{i=-1}^{1} \sum_{k \in \mathbb{Z}}\left(\left\|\hat{u}_{k}\right\|_{L^{4}\left(I, L^{1}\right)}\left\|\hat{v}_{k+i}\right\|_{L^{4}\left(I, \mathrm{M}_{p}^{\lambda}\right)}\right) \\
& \leq \sum_{i=-1}^{1} \sum_{k \in \mathbb{Z}}\left(2^{k\left(\frac{3}{p^{\prime}}+\frac{\lambda}{p}\right)}\left\|\hat{u}_{k}\right\|_{L^{4}\left(I, \mathrm{M}_{p}^{\lambda}\right)}\left\|\hat{v}_{k+i}\right\|_{L^{4}\left(I, \mathrm{M}_{p}^{\lambda}\right)}\right) \\
& \left(\sum_{j \in \mathbb{Z}}\left\|\widehat{I I I}_{j}\right\|_{L^{2}\left(I, \mathrm{M}_{p}^{\lambda}\right)}^{q}\right)^{\frac{1}{q}} \leq \sum_{i=-1}^{1} 2^{-i\left(1-\frac{3}{2} \alpha+\frac{3}{p^{\prime}}+\frac{\lambda}{p}\right)} \sum_{k \in \mathbb{Z}} 2^{k\left(1-\frac{3}{2} \alpha+\frac{3}{p^{\prime}}+\frac{\lambda}{p}\right)}\left\|\hat{u}_{k}\right\|_{L^{4}\left(I, \mathrm{M}_{p}^{\lambda}\right)} \\
& \times 2^{(k+i)\left(1-\frac{3}{2} \alpha+\frac{3}{p^{\prime}}+\frac{\lambda}{p}\right)}\left\|\hat{v}_{k+i}\right\|_{L^{4}\left(I, \mathrm{M}_{p}^{\lambda}\right)} \\
& \leq C\|u\|_{\mathcal{L}^{4}\left(I, \mathcal{F} \dot{\mathcal{N}}_{p, \lambda, q^{\prime}}^{1-\frac{3}{2} \alpha+\frac{3}{p^{\prime}}+\frac{\lambda}{p}}\right)}\|v\| \mathcal{L}^{4}\left(I, \mathcal{F} \dot{\mathcal{N}}_{p, \lambda, q}^{1-\frac{3}{2} \alpha+\frac{3}{p^{\prime}}+\frac{\lambda}{p}}\right) \\
& \leq C\|u\|_{\mathcal{L}^{4}\left(I, \mathcal{F} \mathcal{N}_{p, \lambda, q}^{\frac{3}{2} \alpha-1}\right)}\|v\|_{\mathcal{L}^{4}\left(I, \mathcal{F} \mathcal{N}_{p, \lambda, q}^{\frac{3}{2} \alpha-1}\right)},
\end{aligned}
$$

where we have used the fact $1 \leq q \leq 2$ implies $\mathcal{F N}_{p, \lambda, q}^{\frac{3}{2} \alpha-1} \hookrightarrow \mathcal{F N}_{p, \lambda, q^{\prime}}^{\frac{3}{2} \alpha-1}$ with $q^{\prime}$ is the conjugate of $q$. Estimates (13), (14), (15), (16) and (17) yield (12).

Proposition 12. Let $1 \leq p<\infty, 1 \leq \rho \leq \infty, 1 \leq q \leq 2,0 \leq \lambda<3$ and $\frac{1}{2}<\alpha \leq \frac{2+\frac{3}{p^{\prime}}+\frac{\lambda}{p}}{4-\frac{2}{\rho}}$, and set

$$
X=\mathcal{L}^{\infty}\left([0, \infty), \mathcal{F} \dot{\mathcal{N}}_{p, \lambda, q}^{1-2 \alpha+\frac{3}{p^{\prime}}+\frac{\lambda}{p}}\right) \cap \mathcal{L}^{\rho}\left([0, \infty), \mathcal{F}_{\mathcal{N}^{1}, \lambda, q}^{1-2 \alpha+\frac{3}{p^{\prime}}+\frac{2 \alpha}{\rho}+\frac{\lambda}{p}}\right)
$$

with the norm

$$
\|u\|_{X}=\|u\|_{\mathcal{L}^{\infty}\left([0, \infty), \mathcal{F} \dot{\mathcal{N}}_{p, \lambda, q}^{1-2 \alpha+\frac{3}{p^{\prime}}+\frac{\lambda}{p}}\right)}+\mu\|u\|_{\mathcal{L}^{\rho}\left([0, \infty), \mathcal{F} \dot{\mathcal{N}}_{p, \lambda, q}^{1-2 \alpha+\frac{3}{p^{\prime}}}+\frac{2 \alpha}{p}+\frac{\lambda}{p}\right)} .
$$

There exists a constant $C=C(\alpha, p, q)>0$ depending on $\alpha, p, q$ such that

$$
\|\nabla \cdot(u \otimes v)\|_{\mathcal{L}^{\rho}\left([0, \infty), \mathcal{F} \dot{\mathcal{N}}_{p, \lambda, q}^{1-4 \alpha+\frac{3}{p^{\prime}}+\frac{2 \alpha}{\rho}+\frac{\lambda}{p}}\right)} \leq C \mu^{-1}\|u\|_{X}\|v\|_{X} .
$$

Proof. This proposition is given in [28] for $\frac{1}{2}<\alpha<\frac{2+\frac{3}{p^{\prime}}+\frac{\lambda}{p}}{4-\frac{2}{\rho}}$. For the case $\alpha=\frac{2+\frac{3}{p^{\prime}}+\frac{\lambda}{p}}{4-\frac{2}{\rho}}$ the argument is similar to the method described for (17).

Now, we give an abstract lemma on the existence of fixed point solutions.

Lemma 13. Let $X$ be a Banach space with norm $\|\cdot\|_{X}$ and $B: X \times X \longmapsto X$ be a bounded bilinear operator satisfying

$$
\|B(u, v)\|_{X} \leq \eta\|u\|_{X}\|v\|_{X}
$$

for all $u, v \in X$ and a constant $\eta>0$. Then, if $0<\varepsilon<\frac{1}{4 \eta}$ and if $y \in X$ such that $\|y\|_{X} \leq \varepsilon$, the equation $x:=y+B(x, x)$ has a solution $\bar{x}$ in $X$ such that $\|\bar{x}\|_{X} \leq 2 \varepsilon$. This solution is the only one in the ball $\bar{B}(0,2 \varepsilon)$. Moreover, the solution depends continuously on $y$ in the sense: if $\left\|y^{\prime}\right\|_{X}<\varepsilon, x^{\prime}=y^{\prime}+B\left(x^{\prime}, x^{\prime}\right)$, and $\left\|x^{\prime}\right\|_{X} \leq 2 \varepsilon$, then 


$$
\left\|\bar{x}-x^{\prime}\right\|_{X} \leq \frac{1}{1-4 \varepsilon \eta}\left\|y-y^{\prime}\right\|_{X}
$$

Proof of Theorem 5. We will use Lemma 13 to sketch the proof of the existence results. The functions here are vector fields, whose norm is the sum of the norms of the three components.

For the local existence, we set

$$
Y=\mathcal{L}^{4}\left(I, \mathcal{F} \dot{\mathcal{N}}_{p, \lambda, q}^{1-\frac{3}{2} \alpha+\frac{3}{p^{\prime}}+\frac{\lambda}{p}}\right), I=[0, T) .
$$

Here, as usual, we begin with the mild integral equation

$$
u(t, x)=T_{\Omega, \alpha}(t) u_{0}-\int_{0}^{t} T_{\Omega, \alpha}(t-\tau) \mathbb{P} \nabla \cdot(u \otimes u)(\tau, x) d \tau,
$$

and we consider the bilinear operator $B$ given by

$$
B(u, v)=\int_{0}^{t} T_{\Omega, \alpha}(t-\tau) \mathbb{P} \nabla \cdot(u \otimes v)(\tau) d \tau .
$$

According to Lemma 9 and Proposition 11 we obtain

$$
\begin{aligned}
\|B(u, v)\|_{\mathcal{L}^{4}\left(I, \mathcal{F} \dot{\mathcal{N}}_{p, \lambda, q}^{1-\frac{3}{2} \alpha+\frac{3}{p^{\prime}}+\frac{\lambda}{p}}\right)} & =\left\|\int_{0}^{t} T_{\Omega, \alpha}(t-\tau) \mathbb{P} \nabla \cdot(u \otimes v)(\tau) d \tau\right\|_{\mathcal{L}^{4}\left(I, \mathcal{F} \dot{\mathcal{N}}_{p, \lambda, q}^{1-\frac{3}{2} \alpha+\frac{3}{p^{\prime}}+\frac{\lambda}{p}}\right)} \\
& \leq C\|\nabla \cdot(u \otimes v)\|_{\mathcal{L}^{2}\left(I, \mathcal{F} \dot{\mathcal{N}}_{p, \lambda, q}^{1-3 \alpha+\frac{3}{p^{\prime}}+\frac{\lambda}{p}}\right)} \leq C\|u v\|_{\mathcal{L}^{2}\left(I, \mathcal{F} \dot{\mathcal{N}}_{p, \lambda, q}^{2-3 \alpha+\frac{3}{p^{\prime}}+\frac{\lambda}{p}}\right)} \leq C\|u\|_{Y}\|v\|_{Y} .
\end{aligned}
$$

Lemma 10 yields

$$
\left\|T_{\Omega, \alpha}(t) u_{0}\right\|_{Y} \leq C\left\|u_{0}\right\| \underset{\mathcal{F N}_{p, \lambda, q}^{1-2 \alpha+\frac{3}{p^{\prime}}+\frac{\lambda}{p}}}{ }
$$

Now, we shall decompose the initial data $u_{0}$ into two terms

$$
u_{0}=\mathcal{F}^{-1}\left(\chi_{B(0, \delta)} \hat{u_{0}}\right)+\mathcal{F}^{-1}\left(\chi_{B^{C}(0, \delta)} \hat{u_{0}}\right):=u_{0,1}+u_{0,2},
$$

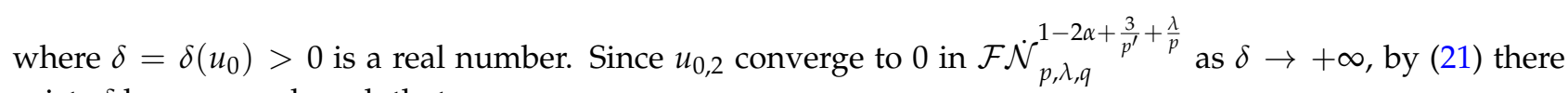
exists $\delta$ large enough such that

$$
\left\|T_{\Omega, \alpha}(t) u_{0,2}\right\|_{Y} \leq \frac{1}{8 C}
$$

For the first term $u_{0,1}$,

$$
\begin{aligned}
\left\|T_{\Omega, \alpha}(t) u_{0,1}\right\|_{Y} & \leq\left\|2^{j\left(1-\frac{3}{2} \alpha+\frac{3}{p^{\prime}}+\frac{\lambda}{p}\right)}\right\| \varphi_{j} e^{-\mu t|\xi|^{2 \alpha}} \chi_{B(0, \delta)} \hat{u}_{0}\left\|_{L^{4}\left(I, \mathrm{M}_{p}^{\lambda}\right)}\right\|_{\ell q} \\
& \leq\left\|2^{j\left(1-\frac{3}{2} \alpha+\frac{3}{p^{\prime}}+\frac{\lambda}{p}\right)}\right\| \sup _{\xi \in B(0, \delta)} e^{-\mu t|\xi|^{2 \alpha}}|\xi|^{\frac{\alpha}{2}}\left\|_{L^{4}([0, T))}\right\| \varphi_{j}|\xi|^{-\frac{\alpha}{2}} \hat{u}_{0}\left\|_{\mathrm{M}_{p}^{\lambda}}\right\|_{\ell^{q}} \\
& \leq C \delta^{\frac{\alpha}{2}} T^{\frac{1}{4}}\left\|u_{0}\right\|_{\mathcal{F}_{\dot{\mathcal{N}}_{p, \lambda, q}}^{1-2 \alpha+\frac{3}{p^{\prime}}+\frac{\lambda}{p}}} .
\end{aligned}
$$

Thus for arbitrary $u_{0}$ in $\mathcal{F} \dot{\mathcal{N}}_{p, \lambda, q}^{1-2 \alpha+\frac{3}{p^{\prime}}+\frac{\lambda}{p}},(20)$ has a unique local solution in $Y$ on $[0, T)$ where

$$
T \leq\left(\frac{1}{8 C^{2} \delta^{\frac{\alpha}{2}}\left\|u_{0}\right\|_{\mathcal{F} \dot{\mathcal{N}}_{p, \lambda, q}^{1-2 \alpha+\frac{3}{p^{\prime}}}+\frac{\lambda}{p}}}\right)^{4} .
$$


For the global existence, we will again use Lemma 13 to ensure the existence of global mild solution with small initial data in the Banach space $X$ given by

$$
X=\mathcal{L}^{\infty}\left([0, \infty), \mathcal{F}_{\mathcal{N}^{\prime}, \lambda, q}^{1-2 \alpha+\frac{3}{p^{\prime}}+\frac{\lambda}{p}}\right) \cap \mathcal{L}^{1}\left([0, \infty), \mathcal{F} \dot{\mathcal{N}}_{p, \lambda, q}^{1+\frac{3}{p^{\prime}}+\frac{\lambda}{p}}\right)
$$

According to Lemma 9 and Proposition 12, we obtain

$$
\begin{aligned}
\|B(u, v)\|_{\mathcal{L}^{1}\left([0, \infty), \mathcal{F} \dot{\mathcal{N}}_{p, \lambda, q}^{1+\frac{3}{p^{p}}+\frac{\lambda}{p}}\right)} & =\left\|\int_{0}^{t} T_{\Omega, \alpha}(t-\tau) \mathbb{P} \nabla \cdot(u \otimes v)(\tau) d \tau\right\|_{\mathcal{L}^{1}\left([0, \infty), \mathcal{F} \dot{\mathcal{N}}_{p, \lambda, q}^{1+\frac{3}{p^{1}}+\frac{\lambda}{p}}\right)} \\
& \leq C\|\nabla \cdot(u \otimes v)\|_{\mathcal{L}^{1}\left([0, \infty), \mathcal{F} \dot{\mathcal{N}}_{p, \lambda, q}^{1-2 \alpha+\frac{3}{p^{\prime}}+\frac{\lambda}{p}}\right)} \leq C \mu^{-1\|u\|_{X}\|v\|_{X} .}
\end{aligned}
$$

Similarly,

$$
\begin{aligned}
\|B(u, v)\|_{\mathcal{L}^{\infty}\left([0, \infty), \mathcal{F} \dot{\mathcal{N}}_{p, \lambda, q}^{1-2 \alpha+\frac{3}{p^{\prime}}+\frac{\lambda}{p}}\right)} & =\left\|\int_{0}^{t} T_{\Omega, \alpha}(t-\tau) \mathbb{P} \nabla \cdot(u \otimes v)(\tau) d \tau\right\|_{\mathcal{L}^{\infty}\left([0, \infty), \mathcal{F} \dot{\mathcal{N}}_{p, \lambda, q}^{1-2 \alpha+\frac{3}{p^{\prime}}+\frac{\lambda}{p}}\right)} \\
& \leq C\|\nabla \cdot(u \otimes v)\| \mathcal{L}^{1}\left([0, \infty), \mathcal{F} \dot{\mathcal{N}}_{p, \lambda, q}^{1-2 \alpha+\frac{3}{p^{\prime}}+\frac{\lambda}{p}}\right) \\
& \leq C \mu^{-1}\|u\|_{X}\|v\|_{X} .
\end{aligned}
$$

Finally,

$$
\|B(u, v)\|_{X} \leq C \mu^{-1}\|u\|_{X}\|v\|_{X}
$$

Lemma 10 yields

$$
\left\|T_{\Omega, \alpha}(t) u_{0}\right\|_{X} \leq C\left\|u_{0}\right\|_{\mathcal{F} \dot{\mathcal{N}}_{p, \lambda, q}^{1-2 \alpha+\frac{3}{p^{\prime}}}+\frac{\lambda}{p}}
$$

If $\left\|u_{0}\right\| \underset{\mathcal{F N}_{p, \lambda, q}^{1-2 \alpha+\frac{3}{p^{1}}+\frac{\lambda}{p}}}{ }<C_{0} \mu$ with $C_{0}=\frac{1}{4 C^{2}}$, then (20) has a unique global solution $u \in X$ satisfying

$$
\|u\|_{X} \leq 2 C\left\|u_{0}\right\| \underset{\mathcal{F} \dot{N}_{p, \lambda, q}^{1-2 \alpha+\frac{3}{p}}+\frac{\lambda}{p}}{ }
$$

This completes the proof of Theorem 5.

\section{The decay property}

In this section, we first present the following interpolation inequalities which have their specific utility in the sequel.

Lemma 14 ([18]). Let $\alpha<\frac{5}{4}, s>\frac{5}{2}-2 \alpha, 1 \leq p, q \leq 2$ and $0 \leq \lambda \leq 3-\frac{3}{2} p$. Then we have

$$
\|u\|_{\mathcal{F N}_{p, \lambda, q}^{1-2 \alpha+\frac{3}{p^{\prime}}+\frac{\lambda}{p}}} \lesssim\|u\|_{L^{2}}^{1-\frac{5 / 2-2 \alpha}{s}}\|u\|_{\dot{H}^{s}}^{\frac{5 / 2-2 \alpha}{s}} .
$$

Lemma 15 ([18]). Let $1 \leq p, q \leq 2$ and $\frac{1}{2}<\alpha \leq 1$. Then we have

$$
\|u v\|_{\dot{H}^{1-\alpha}} \leq C\|u\|_{L^{2}}\|v\|_{\mathcal{F} \dot{\mathcal{N}}_{p, \lambda, q}^{1-\alpha+\frac{3}{p^{\prime}}}+\frac{\lambda}{p}}+C\|u\|_{\dot{H}^{\alpha}}\|v\|_{\mathcal{F N}_{p, \lambda, q}^{1-2 \alpha+\frac{3}{p^{1}}}+\frac{\lambda}{p}} .
$$

Proof. Proof of Theorem 6 In this part, we will focus on the asymptotic behavior of global solutions when $t \rightarrow \infty$, which was developed in different papers such as [11,29,30] and [31, chap. 11]. 
For $k \in \mathbb{N}$, define

$$
\mathcal{A}_{k}=\left\{\xi \in \mathbb{R}^{3} ;|\xi| \leq k \text { and }\left|\hat{u}_{0}(\xi)\right| \leq k\right\} .
$$

Obviously $\mathcal{F}^{-1}\left(\chi_{\mathcal{A}_{k}} \hat{u}_{0}\right)$ converge to $u_{0}$ in $\mathcal{F} \dot{\mathcal{N}}_{p, \lambda, q}^{1-2 \alpha+\frac{3}{p^{\prime}}+\frac{\lambda}{p}}$ as $k \rightarrow+\infty$.

Then, there exists $k \in \mathbb{N}$ such that

$$
\left\|u_{0}-\mathcal{F}^{-1}\left(\chi_{\mathcal{A}_{k}} \hat{u}_{0}\right)\right\|_{\mathcal{F} \dot{\mathcal{N}}_{p, \lambda, q}^{1-2 \alpha+\frac{3}{p^{\prime}}+\frac{\lambda}{p}}} \leq \frac{\varepsilon}{2} .
$$

Put

$$
u_{0, k}=\mathcal{F}^{-1}\left(\chi_{\mathcal{A}_{k}} \hat{u}_{0}\right), w_{0, k}=u_{0}-\mathcal{F}^{-1}\left(\chi_{\mathcal{A}_{k}} \hat{u}_{0}\right) .
$$

Then $u_{0, k} \in \mathcal{F} \dot{\mathcal{N}}_{p, \lambda, q}^{1-2 \alpha+\frac{3}{p^{\prime}}+\frac{\lambda}{p}} \cap \mathrm{L}^{2}$ and

$$
\left\|w_{0, k}\right\|_{\mathcal{F N}_{p, \lambda, q}^{1-2 \alpha+\frac{3}{p^{\prime}}}+\frac{\lambda}{p}} \leq \frac{\varepsilon}{2}
$$

Now, we insert the following system

$$
\left\{\begin{array}{l}
w_{t}+(w \cdot \nabla) w+\mu(-\Delta)^{\alpha} w+\Omega e_{3} \times w+\nabla \pi=0 \quad \text { in }(0,+\infty) \times \mathbb{R}^{3} \\
\nabla \cdot w=0 \\
\left.w\right|_{t=0}=w_{0, k} .
\end{array}\right.
$$

For $\frac{\varepsilon}{2} \leq C_{0} \mu$, we infer from Theorem 5 that the system (24) has a unique global solution such that

$$
w_{k} \in \mathcal{C}\left([0, \infty) ; \mathcal{F N}_{p, \lambda, q}^{1-2 \alpha+\frac{3}{p^{\prime}}+\frac{\lambda}{p}}\right) \cap \mathcal{L}^{1}\left([0, \infty), \mathcal{F N}_{p, \lambda, q}^{1+\frac{3}{p^{\prime}}+\frac{\lambda}{p}}\right)
$$

Moreover, for any $t \geq 0$ we have

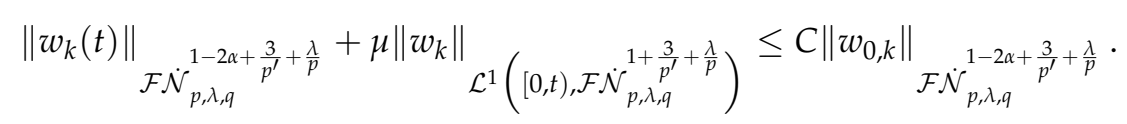

So let's consider the difference $u_{k}=u-w_{k}$, which satisfies

$$
\left\{\begin{array}{l}
\partial_{t} u_{k}+\mu(-\Delta)^{\alpha} u_{k}+\Omega e_{3} \times u_{k}+(u \cdot \nabla) u_{k}+\left(u_{k} \cdot \nabla\right) w_{k}+\nabla\left(\pi-\pi_{k}\right)=0, \\
\nabla \cdot u_{k}=0
\end{array}\right.
$$

where $\pi$ and $\pi_{k}$ are the associated pressures to the solutions $u$ and $w_{k}$, respectively. By taking the inner products with $u_{k}$ and integrating by parts, we can show that

$$
\frac{1}{2} \frac{d}{d t}\left\|u_{k}\right\|_{L^{2}}^{2}+\mu\left\|(-\Delta)^{\frac{\alpha}{2}} u_{k}\right\|_{L^{2}}^{2} \leq\left|\int_{\mathbb{R}^{3}}\left(u_{k} \cdot \nabla\right) w_{k} \cdot u_{k} d x\right|,
$$

where we have used $\Omega\left(e_{3} \times u_{k}\right) \cdot u_{k}=0$.

Integrating by parts, Hölder's inequality and Lemma 15 lead to

$$
\begin{aligned}
\left|\left\langle\nabla \cdot\left(u_{k} \otimes w_{k}\right), u_{k}\right\rangle\right| \leq & \left\|(-\Delta)^{\frac{1}{2}-\frac{\alpha}{2}}\left(u_{k} \otimes w_{k}\right)\right\|_{L^{2}}\left\|(-\Delta)^{\frac{\alpha}{2}} u_{k}\right\|_{L^{2}} \\
\leq & C\left\|u_{k} \otimes w_{k}\right\|_{\dot{H}^{1-\alpha}}\left\|u_{k}\right\|_{\dot{H}^{\alpha}} \leq C\left\|u_{k}\right\|_{L^{2}}\left\|w_{k}\right\|_{\mathcal{F}_{\mathcal{N}_{p, \lambda, q}}^{1-\alpha+\frac{3}{p^{\prime}}+\frac{\lambda}{p}}}\left\|u_{k}\right\|_{\dot{H}^{\alpha}} \\
& +C\left\|u_{k}\right\|_{\dot{H}^{\alpha}}^{2}\left\|w_{k}\right\|_{\mathcal{F}_{\dot{\mathcal{N}}_{p, \lambda, q}}^{1-2 \alpha+\frac{3}{p^{\prime}}+\frac{\lambda}{p}}}
\end{aligned}
$$




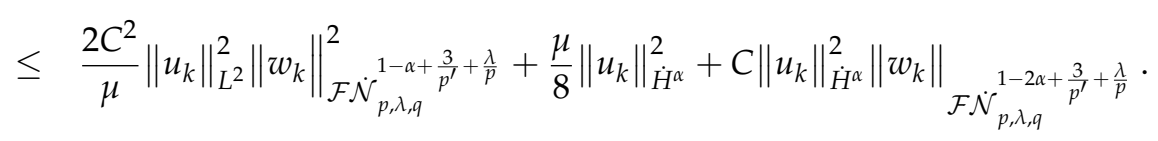

By (23) and (25) we have $\left\|w_{k}\right\|_{\mathcal{F}_{\mathcal{N}}{ }_{p, \lambda, q}^{1-2 \alpha+\frac{3}{p^{\prime}}+\frac{\lambda}{p}}} \leq C \frac{\varepsilon}{2}$. In addition, we assume $\varepsilon$ small enough such that $C^{2} \varepsilon \leq \frac{\mu}{4}$, thus

$$
\left|\left\langle\nabla \cdot\left(u_{k} \otimes w_{k}\right), u_{k}\right\rangle\right| \leq \frac{2 C^{2}}{\mu}\left\|u_{k}\right\|_{L^{2}}^{2}\left\|w_{k}\right\|_{\mathcal{F}_{\mathcal{N}_{p, \lambda, q}}^{1-\alpha+\frac{3}{p^{\prime}}}+\frac{\lambda}{p}}^{2}+\frac{\mu}{4}\left\|u_{k}\right\|_{\dot{H}^{\alpha}}^{2}
$$

We conclude that

$$
\frac{d}{d t}\left\|u_{k}\right\|_{L^{2}}^{2}+\mu\left\|u_{k}\right\|_{\dot{H}^{\alpha}}^{2} \leq \frac{8 C^{2}}{\mu}\left\|u_{k}\right\|_{L^{2}}^{2}\left\|w_{k}\right\|_{\mathcal{F N}_{p, \lambda, q}^{1-\alpha+\frac{3}{p^{\prime}}}}^{2}+\frac{\lambda}{p}
$$

Gronwall's inequality leads to

$$
\left\|u_{k}\right\|_{L^{2}}^{2}+\mu \int_{0}^{t}\left\|u_{k}\right\|_{\dot{H}^{\alpha}}^{2} \leq\left\|u_{0, k}\right\|_{L^{2}}^{2} \exp \left\{\frac{8 C^{2}}{\mu} \int_{0}^{t}\left\|w_{k}\right\|_{\mathcal{F N}_{p, \lambda, q}^{1-\alpha+\frac{3}{p^{\prime}}+\frac{\lambda}{p}}}^{2}\right\} .
$$

Since $q \leq 2$, by Hölder's inequality, we get

$$
\begin{aligned}
& \int_{0}^{t}\left\|w_{k}\right\|_{\mathcal{F N}_{p, \lambda, q}^{1-\alpha+\frac{3}{p^{\prime}}}+\frac{\lambda}{p}}^{2} \leq\left\{\sum_{j \in \mathbb{Z}} 2^{j\left(1-\alpha+\frac{3}{p^{\prime}}+\frac{\lambda}{p}\right) q}\left(\int_{0}^{t}\left\|\varphi_{j} \hat{w}_{k}\right\|_{\mathrm{M}_{p}^{\lambda}}^{2}\right)^{\frac{q}{2}}\right\}^{2 / q} \\
& \leq\left\{\sum_{j \in \mathbb{Z}} 2^{j\left(1-2 \alpha+\frac{3}{p^{\prime}}+\frac{\lambda}{p}\right) \frac{q}{2}} 2^{j\left(1+\frac{3}{p^{\prime}}+\frac{\lambda}{p}\right) \frac{q}{2}}\left\|\varphi_{j} \hat{w}_{k}\right\|_{L^{\infty}\left([0, t), \mathrm{M}_{p}^{\lambda}\right)}^{\frac{q}{2}}\left\|\varphi_{j} \hat{w}_{k}\right\|_{L^{1}\left([0, t), \mathrm{M}_{p}^{\lambda}\right)}^{\frac{q}{2}}\right\}^{2 / q} \\
& \leq\left\|w_{k}\right\|_{\mathcal{L}^{\infty}\left([0, t), \mathcal{F} \dot{\mathcal{N}}_{p, \lambda, q}^{1-2 \alpha+\frac{3}{p^{\prime}}+\frac{\lambda}{p}}\right)}\left\|w_{k}\right\|_{\mathcal{L}^{1}\left([0, t), \mathcal{F} \dot{\mathcal{N}}_{p, \lambda, q}^{1+\frac{3}{p^{\prime}}+\frac{\lambda}{p}}\right)} \\
& \leq \frac{1}{2 \mu}\left(\left\|w_{k}\right\|_{\mathcal{L}^{\infty}\left([0, t), \mathcal{F} \dot{\mathcal{N}}_{p, \lambda, q}^{1-2 \alpha+\frac{3}{p^{\prime}}+\frac{\lambda}{p}}\right)}+\mu\left\|w_{k}\right\|_{\mathcal{L}^{1}\left([0, t), \mathcal{F} \dot{\mathcal{N}}_{p, \lambda, q}^{1+\frac{3}{p^{2}}+\frac{\lambda}{p}}\right.}\right)^{2} \\
& \leq \frac{C^{2}}{2 \mu}\left\|w_{0, k}\right\|_{\mathcal{F N}_{p, \lambda, q}^{1-2 \alpha+\frac{3}{p^{t}}+\frac{\lambda}{p}}}^{2}
\end{aligned}
$$

where we have used (25). Consequently

$$
\left\|u_{k}\right\|_{L^{2}}^{2}+\mu \int_{0}^{t}\left\|u_{k}\right\|_{\dot{H}^{\alpha}}^{2} \leq\left\|u_{0, k}\right\|_{L^{2}}^{2} \exp \left\{\frac{4 C^{4}}{\mu^{2}}\left\|w_{0, k}\right\|_{\mathcal{F}_{\dot{\mathcal{N}}_{p, \lambda, q}}^{1-2 \alpha+\frac{3}{p^{\prime}}}+\frac{\lambda}{p}}^{2}\right\}
$$

Using Lemma 14 with $s=\alpha$ we obtain

$$
\int_{0}^{\infty}\left\|u_{k}\right\|_{\mathcal{F}_{\dot{\mathcal{N}}_{p, \lambda, q}}^{1-2 \alpha+\frac{3}{p^{\prime}}+\frac{\lambda}{p}}}^{\frac{4 \alpha}{5-4 \alpha}} \lesssim C^{\frac{4 \alpha}{5-4 \alpha}} \mu^{-1}\left\|u_{0, k}\right\|_{L^{2}}^{\frac{4 \alpha}{5-4 \alpha}} \times \exp \left\{\frac{8 C^{4} \alpha}{\mu^{2}(5-4 \alpha)}\left\|w_{0, k}\right\|_{\mathcal{F}_{\mathcal{N}_{p, \lambda, q}}^{1-2 \alpha+\frac{3}{p^{1}}+\frac{\lambda}{p}}}^{2}\right\}
$$

The continuity of $u_{k}$ in $\mathcal{F} \dot{\mathcal{N}}_{p, \lambda, q}^{1-2 \alpha+\frac{3}{p^{\prime}}+\frac{\lambda}{p}}$ ensure that there exists a time $t_{0}$ such that

$$
\left\|u_{k}\left(t_{0}\right)\right\|_{\mathcal{F N}_{p, \lambda, q}^{1-2 \alpha+\frac{3}{p^{\prime}}}+\frac{\lambda}{p}} \leq \frac{\varepsilon}{2} .
$$


Then we have

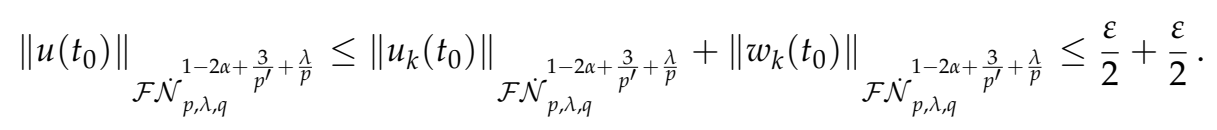

Now, we consider the fractional Navier-Stokes equations with Coriolis forces starting at $t=t_{0}$

$$
\left\{\begin{array}{l}
u_{t}+u \cdot \nabla u+\mu(-\Delta)^{\alpha} u+\Omega e_{3} \times u+\nabla \pi=0 \\
\nabla \cdot u=0 \\
u\left(t_{0}, x\right)=u\left(t_{0}\right)
\end{array}\right.
$$

By Theorem 5 and using the method mentioned in the proof of (25), we directly obtain

$$
\|u(t)\|_{\mathcal{F} \dot{\mathcal{N}}_{p, \lambda, q}^{1-2 \alpha+\frac{3}{p^{\prime}}+\frac{\lambda}{p}}+\mu\|u\|} \underset{\mathcal{L}^{1}\left(\left[t_{0}, t\right), \mathcal{F} \dot{\mathcal{N}}_{p, \lambda, q}^{1+\frac{3}{p^{\prime}}+\frac{\lambda}{p}}\right)}{ } \leq C\left\|u\left(t_{0}\right)\right\|_{\mathcal{F N}_{p, \lambda, q}^{1-2 \alpha+\frac{3}{p^{\prime}}}+\frac{\lambda}{p}} \leq C \mathcal{E}
$$

for all $t \geq t_{0}$. We have completed the proof of Theorem 6 .

\section{Stability of global solutions}

In this section we prove Theorem 7. Let $T^{*}$ be the maximal existence time of a solution $u$ of (1) in $\mathcal{L}^{\infty}\left(\left[0, T^{*}\right) ; \mathcal{F} \dot{\mathcal{N}}_{p, \lambda, q}^{1-2 \alpha+\frac{3}{p^{\prime}}+\frac{\lambda}{p}}\right) \cap \mathcal{L}^{1}\left(\left[0, T^{*}\right), \mathcal{F} \dot{\mathcal{N}}_{p, \lambda, q}^{1+\frac{3}{p^{\prime}}+\frac{\lambda}{p}}\right)$.

In order to prove a blow-up criterion of the solution given by Theorem 5, assume that $T^{*}<\infty$ and $\|u\|$

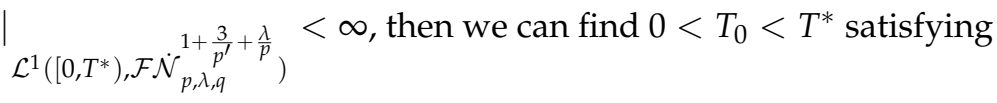

$$
\|u\|_{\mathcal{L}^{1}\left(\left[T_{0}, T^{*}\right), \mathcal{F} \dot{\mathcal{N}}_{p, \lambda, q}^{1+\frac{3}{p^{p}}+\frac{\lambda}{p}}\right)}<\frac{1}{2}
$$

For $t \in\left[T_{0}, T^{*}\right)$, we explicitly consider the integral equation

$$
u(t)=T_{\Omega, \alpha}(t) u\left(T_{0}\right)-\int_{T_{0}}^{t} T_{\Omega, \alpha}(t-s) \mathbb{P} \nabla \cdot(u \otimes u)(s) d s,
$$

we obtain

$$
|\widehat{u}(t, \xi)| \leq e^{-\mu|\xi|^{2 \alpha}}\left|\widehat{u}\left(T_{0}, \xi\right)\right|+\int_{T_{0}}^{t} e^{-\mu(t-s)|\xi|^{2 \alpha}}|\mathbb{P} \nabla \cdot(u \otimes u)(s, \xi)| \mathrm{d} s .
$$

The same reasoning as in the proof of Proposition 12 gives

$$
\|u\|_{\mathcal{L}^{\infty}\left(\left[T_{0}, t\right), \mathcal{F} \dot{\mathcal{N}}_{p, \lambda, q}^{1-2 \alpha+\frac{3}{p^{\prime}}+\frac{\lambda}{p}}\right)} \lesssim\left\|u\left(T_{0}\right)\right\|_{\mathcal{F}_{\mathcal{N}_{p, \lambda, q}}^{1-2 \alpha+\frac{3}{p^{\prime}}}+\frac{\lambda}{p}}+\|u\|_{\mathcal{L}^{\infty}\left(\left[T_{0}, t\right), \mathcal{F} \dot{\mathcal{N}}_{p, \lambda, q}^{1-2 \alpha+\frac{3}{p^{\prime}}}+\frac{\lambda}{p}\right.}\|u\|_{\mathcal{L}^{1}\left(\left[T_{0}, t\right), \mathcal{F} \dot{\mathcal{N}}_{p, \lambda, q}^{1+\frac{3}{p^{\prime}}}+\frac{\lambda}{p}\right.} .
$$

It follows that

$$
\|u\|_{\mathcal{L}^{\infty}\left(\left[T_{0}, t\right), \mathcal{F} \dot{\mathcal{N}}_{p, \lambda, q}^{1-2 \alpha+\frac{3}{p^{\prime}}+\frac{\lambda}{p}}\right)} \lesssim\left\|u\left(T_{0}\right)\right\|_{\mathcal{F N}_{p, \lambda, q}^{1-2 \alpha+\frac{3}{p^{\prime}}+\frac{\lambda}{p}}}+\frac{1}{2}\|u\|_{\mathcal{L}^{\infty}\left(\left[T_{0}, t\right), \mathcal{F} \dot{\mathcal{N}}_{p, \lambda, q}^{1-2 \alpha+\frac{3}{p^{\prime}}+\frac{\lambda}{p}}\right)} .
$$

We can deduce that

$$
\sup _{T_{0} \leq s \leq t}\|u\|_{\mathcal{F} \dot{\mathcal{N}}_{p, \lambda, q}^{1-2 \alpha+\frac{3}{p^{\prime}}+\frac{\lambda}{p}}} \lesssim 2\left\|u\left(T_{0}\right)\right\|_{\mathcal{F N}_{p, \lambda, q}^{1-2 \alpha+\frac{3}{p^{\prime}}+\frac{\lambda}{p}}}, \forall t \in\left[T_{0}, T^{*}\right)
$$


Setting

$$
M=\max \left(2\left\|u\left(T_{0}\right)\right\|_{\mathcal{F} \dot{\mathcal{N}}_{p, \lambda, q}^{1-2 \alpha+\frac{3}{p^{\prime}}}+\frac{\lambda}{p},} \max _{t \in\left[0, T_{0}\right]}\|u\|_{\mathcal{F} \dot{\mathcal{N}}_{p, \lambda, q}^{1-2 \alpha+\frac{3}{p^{\prime}}}+\frac{\lambda}{p}}\right)
$$

we have

$$
\|u(t)\|_{\mathcal{F} \dot{\mathcal{N}}_{p, \lambda, q}^{1-2 \alpha+\frac{3}{p^{\prime}}+\frac{\lambda}{p}}} \lesssim M, \forall t \in\left[0, T^{*}\right) .
$$

On the other side

$$
u(t)=e^{-t \mu(-\Delta)^{\alpha}} u_{0}-\Omega \int_{0}^{t} e^{-\mu(t-\tau)(-\Delta)^{\alpha}} \mathbb{P}\left(e_{3} \times u\right)(\tau) d \tau-\int_{0}^{t} e^{-\mu(t-\tau)(-\Delta)^{\alpha}} \mathbb{P} \nabla \cdot(u \otimes u)(\tau) d \tau .
$$

Then

$$
\begin{aligned}
u\left(t^{\prime}\right)-u(t)= & \left(e^{-\mu t^{\prime}(-\Delta)^{\alpha}} u_{0}-e^{-\mu t(-\Delta)^{\alpha}} u_{0}\right) \\
& -\left(\int_{0}^{t^{\prime}} e^{-\mu\left(t^{\prime}-\tau\right)(-\Delta)^{\alpha}} \mathbb{P} \nabla \cdot(u \otimes u)(\tau) d \tau-\int_{0}^{t} e^{-\mu(t-\tau)(-\Delta)^{\alpha}} \mathbb{P} \nabla \cdot(u \otimes u)(\tau) d \tau\right) \\
& \left.-\Omega\left(\int_{0}^{t^{\prime}} e^{-\mu\left(t^{\prime}-\tau\right)(-\Delta)^{\alpha}} \mathbb{P}\left(e_{3} \times u\right)(\tau) d \tau-\int_{0}^{t} e^{-\mu(t-\tau)(-\Delta)^{\alpha}} \mathbb{P}\left(e_{3} \times u\right)\right)(\tau) d \tau\right) \\
= & {\left[e^{-\mu t^{\prime}(-\Delta)^{\alpha}} u_{0}-e^{-\mu t(-\Delta)^{\alpha}} u_{0}\right]-\left[\int_{t}^{t^{\prime}} e^{-\mu\left(t^{\prime}-\tau\right)(-\Delta)^{\alpha}} \mathbb{P} \nabla \cdot(u \otimes u)(\tau) d \tau\right] } \\
& -\left[\int_{0}^{t} e^{-\mu(t-\tau)(-\Delta)^{\alpha}}\left(e^{-\mu\left(t^{\prime}-t\right)(-\Delta)^{\alpha}}-1\right) \mathbb{P} \nabla \cdot(u \otimes u)(\tau) d \tau\right] \\
& -\Omega\left[\int_{t}^{t^{\prime}} e^{-\mu\left(t^{\prime}-\tau\right)(-\Delta)^{\alpha}} \mathbb{P}\left(e_{3} \times u\right)(\tau) d \tau\right] \\
& -\Omega\left[\int_{0}^{t} e^{-\mu(t-\tau)(-\Delta)^{\alpha}}\left(e^{-\mu\left(t^{\prime}-t\right)(-\Delta)^{\alpha}}-1\right) \mathbb{P}\left(e_{3} \times u\right)(\tau) d \tau\right] \\
:= & J_{1}+J_{2}+J_{3}+J_{4}+J_{5} .
\end{aligned}
$$

We will estimate $J_{1}, J_{2}, J_{3}, J_{4}$ and $J_{5}$;

$$
\begin{aligned}
& \left\|J_{1}\right\| \underset{\mathcal{F N}_{p, \lambda, q}^{1-2 \alpha+\frac{3}{p^{\prime}}+\frac{\lambda}{p}}}{ }=\left\|2^{j\left(1-2 \alpha+\frac{3}{p^{\prime}}+\frac{\lambda}{p}\right)}\right\| \varphi_{j}\left(e^{-\mu t^{\prime}|\xi|^{2 \alpha}}-e^{-\mu t|\xi|^{2 \alpha}}\right) \hat{u}_{0}\left\|_{\mathrm{M}_{p}^{\lambda}}\right\|_{\ell q} \\
& \leq\left\|2^{j\left(1-2 \alpha+\frac{3}{p^{\prime}}+\frac{\lambda}{p}\right)}\right\| \varphi_{j}\left(e^{-\mu\left(t^{\prime}-t\right)|\xi|^{2 \alpha}}-1\right) \hat{u}_{0}\left\|_{\mathrm{M}_{p}^{\lambda}}\right\|_{\ell q^{\prime}}, \\
& \left\|J_{2}\right\|_{\mathcal{F N}_{p, \lambda, q}^{1-2 \alpha+\frac{3}{p^{\prime}}}+\frac{\lambda}{p}} \leq\left\|2^{j\left(1-2 \alpha+\frac{3}{p^{\prime}}+\frac{\lambda}{p}\right)} \int_{t}^{t^{\prime}}\right\| \varphi_{j} e^{-\mu\left(t^{\prime}-\tau\right)|\xi|^{2 \alpha}} \mathcal{F}(\nabla \cdot u \otimes u)(\tau)\left\|_{\mathrm{M}_{p}^{\lambda}} d \tau\right\|_{\ell^{q}} \\
& \leq\left\|2^{j\left(2-2 \alpha+\frac{3}{p^{\prime}}+\frac{\lambda}{p}\right)} \int_{t}^{t^{\prime}}\right\| \varphi_{j} \mathcal{F}(u \otimes u)(\tau)\left\|_{\mathrm{M}_{p}^{\lambda}} d \tau\right\|_{\ell^{\prime}}, \\
& \left\|J_{3}\right\|_{\mathcal{F}_{\dot{\mathcal{N}}_{p, \lambda, q}}^{1-2 \alpha+\frac{3}{p^{\prime}}+\frac{\lambda}{p}}} \leq\left\|2^{j\left(1-2 \alpha+\frac{3}{p^{\prime}}+\frac{\lambda}{p}\right)} \times \int_{0}^{t}\right\| \varphi_{j} e^{-\mu\left(t^{\prime}-\tau\right)|\xi|^{2 \alpha}}\left(1-e^{-\mu\left(t^{\prime}-t\right)|\xi|^{2 \alpha}}\right) \mathcal{F}(\nabla \cdot(u \otimes u)(\tau))\left\|_{\mathrm{M}_{p}^{\lambda}} d \tau\right\|_{\ell q} \\
& \leq\left\|2^{j\left(2-2 \alpha+\frac{3}{p^{\prime}}+\frac{\lambda}{p}\right)} \int_{0}^{t}\right\| \varphi_{j}\left(e^{-\mu\left(t^{\prime}-t\right)|\xi|^{2 \alpha}}-1\right) \mathcal{F}(u \otimes u)(\tau)\left\|_{\mathrm{M}_{p}^{\lambda}} d \tau\right\|_{\ell^{q^{\prime}}}
\end{aligned}
$$




$$
\begin{aligned}
\left\|J_{4}\right\|_{\mathcal{F N}_{\dot{\mathcal{N}}^{1}, \lambda, q}^{1-2 \alpha+\frac{3}{p^{\prime}}+\frac{\lambda}{p}}} & \lesssim\left\|2^{j\left(1-2 \alpha+\frac{3}{p^{\prime}}+\frac{\lambda}{p}\right)} \int_{t}^{t^{\prime}}\right\| \varphi_{j} e^{-\mu\left(t^{\prime}-\tau\right)|\xi|^{2 \alpha}} \mathcal{F}\left(e_{3} \times u\right)(\tau)\left\|_{\mathrm{M}_{p}^{\lambda}} d \tau\right\|_{\ell q} \\
& \lesssim\left\|2^{j\left(1-2 \alpha+\frac{3}{p^{\prime}}+\frac{\lambda}{p}\right)} \int_{t}^{t^{\prime}}\right\| \varphi_{j} \mathcal{F}\left(e_{3} \times u\right)(\tau)\left\|_{\mathrm{M}_{p}^{\lambda}} d \tau\right\|_{\ell q^{\prime}}
\end{aligned}
$$

and

$$
\begin{aligned}
& \left\|J_{5}\right\|_{\mathcal{F} \dot{\mathcal{N}}_{p, \lambda, q}^{1-2 \alpha+\frac{3}{p^{\prime}}+\frac{\lambda}{p}}} \lesssim\left\|2^{j\left(1-2 \alpha+\frac{3}{p^{\prime}}+\frac{\lambda}{p}\right)} \times \int_{0}^{t}\right\| \varphi_{j} e^{-\mu\left(t^{\prime}-\tau\right)|\xi|^{2 \alpha}}\left(1-e^{-\mu\left(t^{\prime}-t\right)|\xi|^{2 \alpha}}\right) \mathcal{F}\left(e_{3} \times u(\tau)\right)\left\|_{\mathrm{M}_{p}^{\lambda}} d \tau\right\|_{\ell q} \\
& \lesssim\left\|2^{j\left(1-2 \alpha+\frac{3}{p^{\prime}}+\frac{\lambda}{p}\right)} \int_{0}^{t}\right\| \varphi_{j}\left(e^{-\mu\left(t^{\prime}-t\right)|\xi|^{2 \alpha}}-1\right) \mathcal{F}\left(e_{3} \times u\right)(\tau)\left\|_{\mathrm{M}_{p}^{\lambda}} d \tau\right\|_{\ell q} .
\end{aligned}
$$

The dominated convergence theorem gives

$$
\limsup _{t, t^{\prime} \succ T^{*}, t \leq t^{\prime}}\left\|u(t)-u\left(t^{\prime}\right)\right\|_{\mathcal{F N}_{p, \lambda, q}^{1-2 \alpha+\frac{3}{p^{\prime}}+\frac{\lambda}{p}}}=0
$$

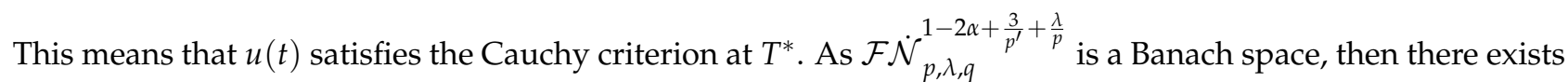
an element $u^{*}$ in $\mathcal{F} \dot{\mathcal{N}}_{p, \lambda, q}^{1-2 \alpha+\frac{3}{p^{\prime}}+\frac{\lambda}{p}}$ such that $u(t) \rightarrow u^{*}$ in $\mathcal{F N}_{p, \lambda, q}^{1-2 \alpha+\frac{3}{p^{+}}+\frac{\lambda}{p}}$ as $t \rightarrow T^{*}$. Set $u\left(T^{*}\right)=u^{*}$ and consider the fractional Navier-Stokes equations with Coriolis force starting by $u^{*}$. By the well-posedness we obtain a solution existing on a larger time interval than $\left[0, T^{*}\right)$, which is a contradiction.

Now, let $v \in \mathcal{C}\left(\left[0, T^{*}\right) ; \mathcal{F N}_{p, \lambda, q}^{1-2 \alpha+\frac{3}{p^{\prime}}+\frac{\lambda}{p}}\right) \cap \mathcal{L}^{1}\left(\left[0, T^{*}\right), \mathcal{F} \dot{\mathcal{N}}_{p, \lambda, q}^{1+\frac{3}{p^{\prime}}+\frac{\lambda}{p}}\right)$ be the maximal solution of (1) corresponding to the initial condition $v_{0}$. We want to prove $T^{*}=\infty$.

Put $w=v-u$ and $w_{0}=v_{0}-u_{0}$. We have

$$
w_{t}+\mu(-\Delta)^{\alpha} w+\Omega e_{3} \times w+w \cdot \nabla w+u \cdot \nabla w+w \cdot \nabla u=-\nabla \pi .
$$

We first apply $\mathbb{P}$ to the above equation, then we have

$$
w_{t}+\mu(-\Delta)^{\alpha} w=-\Omega \mathbb{P} e_{3} \times w-\mathbb{P} \nabla \cdot(w \otimes w)-\mathbb{P} \nabla \cdot(u \otimes w)-\mathbb{P} \nabla \cdot(w \otimes u) .
$$

Due to Duhamel's formula, we write

$$
\begin{aligned}
|\widehat{w}(t, \xi)| \leq & e^{-\mu|\xi|^{2 \alpha} t}|\widehat{w}(0, \xi)|+\int_{0}^{t} e^{-\mu(t-s)|\xi|^{2 \alpha}}|\mathcal{F}(\mathbb{P} \nabla \cdot(w \otimes w))(s, \xi)| \mathrm{d} s \\
& +\int_{0}^{t} e^{-\mu(t-s)|\xi|^{2 \alpha}}|\mathcal{F}(\mathbb{P} \nabla \cdot(u \otimes w))(s, \xi)| \mathrm{d} s \\
& +\int_{0}^{t} e^{-\mu(t-s)|\xi|^{2 \alpha}}|\mathcal{F}(\mathbb{P} \nabla \cdot(w \otimes u))(s, \xi)| \mathrm{d} s \\
& +|\Omega| \int_{0}^{t} e^{-\mu(t-s)|\xi|^{2 \alpha}}\left|\mathcal{F}\left(\mathbb{P} e_{3} \times w\right)(s, \xi)\right| \mathrm{d} s
\end{aligned}
$$

Then, for $t \in\left[0, T^{*}\right)$ we get

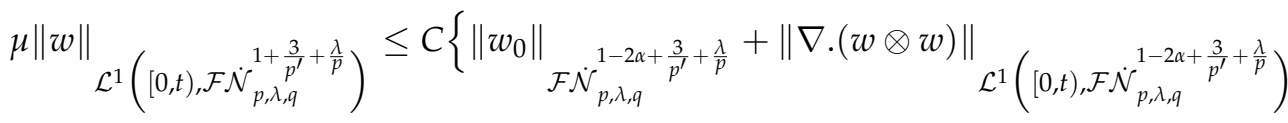

$$
\begin{aligned}
& +\|\nabla \cdot(u \otimes w)\| \mathcal{L}^{1}\left([0, t), \mathcal{F} \dot{\mathcal{N}}_{p, \lambda, q}^{1-2 \alpha+\frac{3}{p^{\prime}}+\frac{\lambda}{p}}\right) \\
& +\|\nabla \cdot(w \otimes u)\| \mathcal{L}^{1}\left([0, t), \mathcal{F} \dot{\mathcal{N}}_{p, \lambda, q}^{1-2 \alpha+\frac{3}{p^{\prime}}+\frac{\lambda}{p}}\right) \\
& \left.+|\Omega|\left\|e_{3} \times w\right\|_{\mathcal{L}^{1}\left([0, t), \mathcal{F} \dot{\mathcal{N}}_{p, \lambda, q}^{1-2 \alpha+\frac{3}{p^{\prime}}+\frac{\lambda}{p}}\right)}\right\} .
\end{aligned}
$$


Similarly,

$$
\begin{aligned}
& \|w\|_{\mathcal{L}^{\infty}\left([0, t), \mathcal{F} \dot{\mathcal{N}}_{p, \lambda, q}^{1-2 \alpha+\frac{3}{p^{\prime}}+\frac{\lambda}{p}}\right)} \leq\left\|w_{0}\right\|_{\mathcal{F N}_{p, \lambda, q}^{1-2 \alpha+\frac{3}{p^{\prime}}+\frac{\lambda}{p}}+\|\nabla \cdot(w \otimes w)\|_{\mathcal{L}^{1}}\left([0, t), \mathcal{F} \dot{\mathcal{N}}_{p, \lambda, q}^{1-2 \alpha+\frac{3}{p^{\prime}}+\frac{\lambda}{p}}\right)} \\
& +\|\nabla \cdot(u \otimes w)\|_{\mathcal{L}^{1}\left([0, t), \mathcal{F} \dot{\mathcal{N}}_{p, \lambda, q}^{1-2 \alpha+\frac{3}{p^{1}}+\frac{\lambda}{p}}\right)} \\
& +\|\nabla \cdot(w \otimes u)\|_{\mathcal{L}^{1}\left([0, t), \mathcal{F} \dot{\mathcal{N}}_{p, \lambda, q}^{1-2 \alpha+\frac{3}{p^{1}}+\frac{\lambda}{p}}\right)} \\
& +|\Omega|\left\|e_{3} \times w\right\|_{\mathcal{L}^{1}\left([0, t), \mathcal{F} \dot{\mathcal{N}}_{p, \lambda, q}^{1-2 \alpha+\frac{3}{p^{\prime}}+\frac{\lambda}{p}}\right)} .
\end{aligned}
$$

Consequently, for $t \in\left[0, T^{*}\right)$ we get

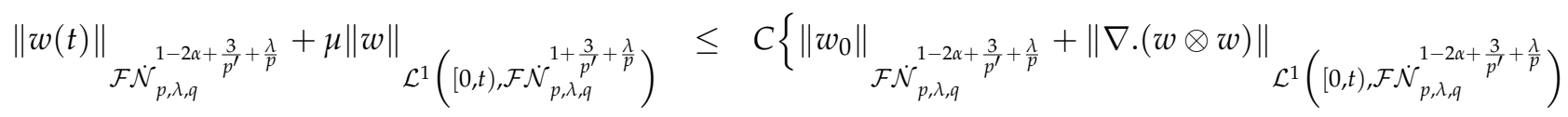

$$
\begin{aligned}
& +\|\nabla \cdot(u \otimes w)\| \mathcal{L}^{1}\left([0, t), \mathcal{F} \dot{\mathcal{N}}_{p, \lambda, q}^{1-2 \alpha+\frac{3}{p^{\prime}}+\frac{\lambda}{p}}\right) \\
& +\|\nabla \cdot(w \otimes u)\|_{\mathcal{L}^{1}\left([0, t), \mathcal{F} \dot{\mathcal{N}}_{p, \lambda, q}^{1-2 \alpha+\frac{3}{p^{\prime}}+\frac{\lambda}{p}}\right)} \\
& \left.+|\Omega|\left\|e_{3} \times w\right\| \mid \mathcal{L}^{1}\left([0, t), \mathcal{F} \dot{\mathcal{N}}_{p, \lambda, q}^{1-2 \alpha+\frac{3}{p^{\prime}}+\frac{\lambda}{p}}\right)\right\} \\
& \lesssim\left\|w_{0}\right\|_{\mathcal{F N}_{p, \lambda, q}^{1-2 \alpha+\frac{3}{p^{\prime}}+\frac{\lambda}{p}}}+L_{1}+L_{2}+L_{3} \text {. }
\end{aligned}
$$

where

$$
\begin{aligned}
& L_{1}=\|\nabla \cdot(w \otimes w)\| \mathcal{L}_{\mathcal{L}^{1}\left([0, t), \mathcal{F} \dot{\mathcal{N}}_{p, \lambda, q}^{1-2 \alpha+\frac{3}{p^{\prime}}+\frac{\lambda}{p}}\right)}, \\
& L_{2}=\|\nabla \cdot(u \otimes w)\|{ }_{\mathcal{L}^{1}\left([0, t), \mathcal{F} \dot{\mathcal{N}}_{p, \lambda, q}^{1-2 \alpha+\frac{3}{p^{\prime}}+\frac{\lambda}{p}}\right)}+\|\nabla \cdot(w \otimes u)\| \|_{\mathcal{L}^{1}\left([0, t), \mathcal{F} \dot{\mathcal{N}}_{p, \lambda, q}^{1-2 \alpha+\frac{3}{p^{\prime}}+\frac{\lambda}{p}}\right)}
\end{aligned}
$$

and $L_{3}=|\Omega|\left\|e_{3} \times w\right\|_{\mathcal{L}^{1}\left([0, t), \mathcal{F} \dot{\mathcal{N}}_{p, \lambda, q}^{1-2 \alpha+\frac{3}{p^{\prime}}+\frac{\lambda}{p}}\right)}$.

The same calculus as in the proof of Proposition 12 gives

$$
\begin{aligned}
& \left.L_{1} \lesssim\|w\|_{\mathcal{L}^{\infty}\left([0, t), \mathcal{F} \dot{\mathcal{N}}_{p, \lambda, q}^{1-2 \alpha+\frac{3}{p^{\prime}}+\frac{\lambda}{p}}\right)}\|w\|_{\mathcal{L}^{1}\left([0, t), \mathcal{F} \dot{\mathcal{N}}_{p, \lambda, q}^{1+\frac{3}{p^{1}}+\frac{\lambda}{p}}\right.}\right)^{\prime}
\end{aligned}
$$

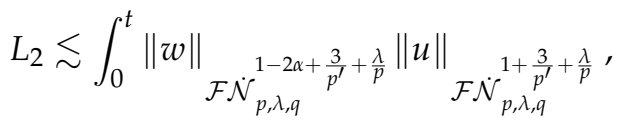

$$
\begin{aligned}
& L_{3} \lesssim|\Omega|\|w\|_{\mathcal{L}^{1}\left([0, t), \mathcal{F} \dot{\mathcal{N}}_{p, \lambda, q}^{1-2 \alpha+\frac{3}{p^{\prime}}+\frac{\lambda}{p}}\right)} .
\end{aligned}
$$

Then

$$
\begin{aligned}
& \|w(t)\|{\mathcal{F} \dot{\mathcal{N}}_{p, \lambda, q}^{1-2 \alpha+\frac{3}{p^{\prime}}+\frac{\lambda}{p}}+\mu\|w\|}_{\mathcal{L}^{1}\left([0, t), \mathcal{F} \dot{\mathcal{N}}_{p, \lambda, l, q}^{1+\frac{3}{p^{\prime}}+\frac{\lambda}{p}}\right)}
\end{aligned}
$$

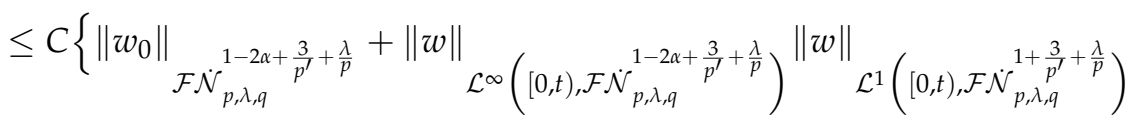




Put

$$
T=\sup \left\{t \in\left[0, T^{*}\right),\|w\|_{\mathcal{L}^{\infty}\left([0, t], \mathcal{F} \dot{\mathcal{N}}_{p, \lambda, q}^{1-2 \alpha+\frac{3}{p^{\prime}}+\frac{\lambda}{p}}\right)}<\frac{\mu}{4 C}\right\}
$$

For $t \in[0, T)$, we have

$$
\begin{aligned}
& \left.\|w(t)\|_{\mathcal{F} \dot{\mathcal{N}}_{p, \lambda, q}^{1-2 \alpha+\frac{3}{p^{\prime}}+\frac{\lambda}{p}}}+\frac{\mu}{2}\|w\|_{\mathcal{L}^{1}\left([0, t), \mathcal{F} \dot{\mathcal{N}}_{p, \lambda, q}^{1-2 \alpha+\frac{3}{p^{\prime}}}+\frac{\lambda}{p}\right.}\right)
\end{aligned}
$$

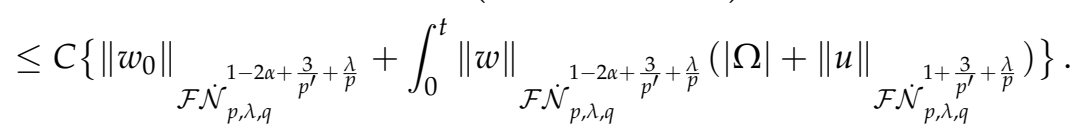

Gronwall's Lemma yields

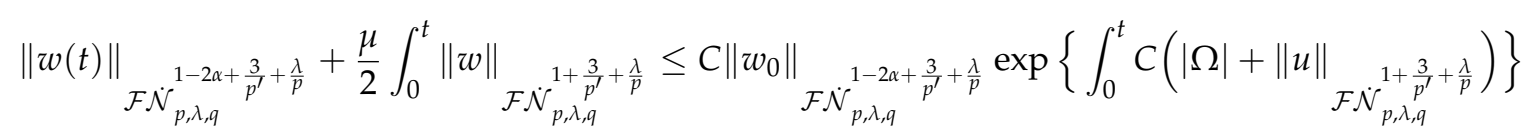

$$
\begin{aligned}
& \leq C\left\|w_{0}\right\|_{\mathcal{F N}_{\mathcal{N}_{p, \lambda, q}}^{1-2 \alpha+\frac{3}{p^{\prime}}+\frac{\lambda}{p}}} \exp \left\{\int_{0}^{\infty} C\left(|\Omega|+\|u\|_{\mathcal{F N}_{p, \lambda, q}^{1+\frac{3}{p^{\prime}}+\frac{\lambda}{p}}}\right)\right\}
\end{aligned}
$$

Thus if we take $C_{0}$ sufficiently small in (7), we have

$$
\|w(t)\|_{\mathcal{F}_{\mathcal{N}}{ }_{p, \lambda, q}^{1-2 \alpha+\frac{3}{p^{\prime}}+\frac{\lambda}{p}}}+\frac{\mu}{2}\|w\|_{\mathcal{L}^{1}\left([0, t), \mathcal{F} \dot{\mathcal{N}}_{p, \lambda, q}{ }^{1-2 \alpha+\frac{3}{p^{\prime}}+\frac{\lambda}{p}}\right)}<\frac{\mu}{8 \mathrm{C}},
$$

which contradicts the definition (30).

Then $T=T^{*}$ and $\|w\|_{\mathcal{L}^{1}\left(\left[0, T^{*}\right), \mathcal{F} \dot{\mathcal{N}}_{p, \lambda, q}^{1+\frac{3}{p^{\prime}}+\frac{\lambda}{p}}\right)}<\infty$, therefore $T^{*}=\infty$. This completes the proof of Theorem 7.

\section{Conclusion}

Using harmonic analysis tools such as decomposition of Littlewood-Paley and the fixed point argument in Banach space, we obtain some results of existence, uniqueness, stability and asymptotic behaviour of solutions of the fractional Navier-Stokes equations with Coriolis force for small initial data. Moreover, local well-posedness results of these equations for large initial data are also discussed. The adopted functional framework is the critical Fourier-Besov-Morrey space $\mathcal{F} \mathcal{N}_{p, \lambda, q}^{1-2 \alpha+\frac{3}{p^{\prime}}+\frac{\lambda}{p}}$ which covers many classical spaces, e.g. the Fourier-Herz space $\dot{\mathcal{B}}_{q}^{1-2 \alpha+\frac{3}{p^{\prime}}+\frac{\lambda}{p}}$, the Fourier-Besov-Lebesgue space $\operatorname{FB}_{p, q}^{1-2 \alpha+\frac{3}{p^{\prime}}+\frac{\lambda}{p}}$ and the Lei-Lin's space $\chi^{1-2 \alpha+\frac{3}{p^{\prime}}+\frac{\lambda}{p}}$. These spaces $\mathcal{F N}_{p, \lambda, q}^{1-2 \alpha+\frac{3}{p^{\prime}}+\frac{\lambda}{p}}$ are some refined functional spaces, more suitable and more adapted for studying these equations.

Acknowledgments: The authors are grateful to the referee and the editorial board for some useful comments that improved the presentation of the paper.

Author Contributions: All authors contributed equally to the writing of this paper. All authors read and approved the final manuscript.

Conflicts of Interest: "The authors declare no conflict of interest."

\section{References}

[1] Majda, A. (2003). Introduction to PDEs and Waves for the Atmosphere and Ocean (Vol. 9). American Mathematical Soc..

[2] Chemin, J. Y., Desjardins, B., Gallagher, I., \& Grenier, E. (2006). Mathematical geophysics, volume 32 of Oxford Lecture Series in Mathematics and its Applications. Oxford University Press, Oxford.

[3] Hieber, M., \& Shibata, Y. (2010). The Fujita-Kato approach to the Navier-Stokes equations in the rotational framework. Mathematische Zeitschrift, 265(2), 481-491.

[4] Chemin, J. Y., Desjardins, B., Gallagher, I., \& Grenier, E. (2002, January). Anisotropy and dispersion in rotating fluids. In Nonlinear partial differential equations and their applications. Collège de France Seminar (Vol. 14, pp. 171-192). 
[5] Iwabuchi, T., \& Takada, R. (2013). Global solutions for the Navier-Stokes equations in the rotational framework. Mathematische Annalen, 357(2), 727-741.

[6] Iwabuchi, T., \& Takada, R. (2014). Global well-posedness and ill-posedness for the Navier-Stokes equations with the Coriolis force in function spaces of Besov type. Journal of Functional Analysis, 267(5), 1321-1337.

[7] Giga, Y., Inui, K., Mahalov, A., \& Matsui, S. Y. (2005). Uniform local solvability for the Navier-Stokes equations with the Coriolis force. Methods and Applications of Analysis, 12(4), 381-394.

[8] Yoshikazu, G. I. G. A., Katsuya, I. N. U. I., Mahalov, A., \& Matsui, S. Y. (2006). Navier-Stokes equations in a rotating frame in $\mathbb{R}^{3}$ with initial data nondecreasing at infinity. Hokkaido mathematical journal, 35(2), 321-364.

[9] Sawada, O. (2004). The Navier-Stokes flow with linearly growing initial velocity in the whole space. Boletim da Sociedade Paranaense de Matemática, 22(2), 75-96.

[10] Wang, W., \& Wu, G. (2018). Global mild solution of the generalized Navier-Stokes equations with the Coriolis force. Applied Mathematics Letters, 76, 181-186.

[11] Benameur, J. (2015). Long time decay to the Lei-Lin solution of 3D NavierÜStokes equations. Journal of Mathematical Analysis and Applications, 422(1), 424-434.

[12] Fang, D., Han, B., \& Hieber, M. (2015). Global Existence Results for the Navier-Stokes Equations in the Rotational Framework in Fourier-Besov Spaces. In Operator Semigroups Meet Complex Analysis, Harmonic Analysis and Mathematical Physics (pp. 199-211). Birkhäuser, Cham.

[13] Konieczny, P., \& Yoneda, T. (2011). On dispersive effect of the Coriolis force for the stationary Navier-Stokes equations. Journal of Differential Equations, 250(10), 3859-3873.

[14] Kato, T. (1992). Strong solutions of the Navier-Stokes equation in Morrey spaces. Boletim da Sociedade Brasileira de Matemática-Bulletin/Brazilian Mathematical Society, 22(2), 127-155.

[15] Taylor, M. E. (1992). Analysis on Morrey spaces and applications to Navier-Stokes and other evolution equations. Communications in Partial Differential Equations, 17(9-10), 1407-1456.

[16] Yuan, W., Sickel, W., \& Yang, D. (2010). Morrey and Campanato Meet Besov, Lizorkin and Triebel. Springer.

[17] Cannone, M., \& Wu, G. (2012). Global well-posedness for NavierÜStokes equations in critical Fourier-Herz spaces. Nonlinear Analysis: Theory, Methods \& Applications, 75(9), 3754-3760.

[18] El Baraka, A., \& Toumlilin, M. (2017). Global well-posedness and decay results for 3D generalized magneto-hydrodynamic equations in critical Fourier-Besov-Morrey spaces. Electronic Journal of Differential Equations, 2017(65), 1-20.

[19] Ito, H., \& Kato, J. (2015). A remark on a priori estimate for the Navier-Stokes equations with the Coriolis force. arXiv preprint arXiv:1512.01814.

[20] Zhao, H., \& Wang, Y. (2017). A remark on the Navier-Stokes equations with the Coriolis force. Mathematical Methods in the Applied Sciences, 40(18), 7323-7332.

[21] Gallagher, I., Iftimie, D., \& Planchon, F. (2003). Asymptotics and stability for global solutions to the Navier-Stokes equations. In Annales de l'Institut fourier (Vol. 53, No. 5, pp. 1387-1424).

[22] Kawanago, T. (1998). Stability estimate for strong solutions of the Navier-Stokes system and its applications. Electronic Journal of Differential Equations,(1998)(15), 1-23.

[23] Ponce, G., Racke, R., Sideris, T. C., \& Titi, E. S. (1994). Global stability of large solutions to the 3D Navier-Stokes equations. Communications in Mathematical Physics, 159(2), 329-341.

[24] Ferreira, L. C., \& Lima, L. S. (2014). Self-similar solutions for active scalar equations in Fourier-Besov-Morrey spaces. Monatshefte für Mathematik, 175(4), 491-509.

[25] Babin, A., Mahalov, A., \& Nicolaenko, B. (1995). Long-time averaged Euler and Navier-Stokes equations for rotating fluids. In Structure and Dynamics of non-linear waves in Fluids, 1994 IUTAM Conference, K. Kirehgässner and A. Mielke (eds), World Scientific (pp. 145-157).

[26] Babin, A., Mahalov, A., \& Nicolaenko, B. (1997). Regularity and integrability of 3D Euler and Navier-Stokes equations for rotating fluids. Asymptotic Analysis, 15(2), 103-150.

[27] Babin, A., Mahalov, A., \& Nicolaenko, B. (1999). Global regularity of 3D rotating Navier-Stokes equations for resonant domains. Indiana University Mathematics Journal, 1133-1176.

[28] El Baraka, A., \& Toumlilin, M. (2017). Global Well-Posedness for Fractional Navier-Stokes Equations in critical Fourier-Besov-Morrey Spaces. Moroccan Journal of Pure and Applied Analysis, 3(1), 1-13.

[29] Gallagher, I., Iftimie, D., \& Planchon, F. (2002). Non-blowup at large times and stability for global solutions to the Navier-Stokes equations. Comptes rendus de l'Académie des Sciences, 334(4), 289-292.

[30] Xiao, W., Chen, J., Fan, D., \& Zhou, X. (2014). Global well-posedness and long time decay of fractional navier-stokes equations in fourier-besov spaces. In Abstract and Applied Analysis (Vol. 2014). Hindawi.

[31] Lemarié-Rieusset, Pierre Gilles, P. G. (2016). The Navier-Stokes problem in the 21st century. Chapman and Hall/CRC. 
(C) 2019 by the authors; licensee PSRP, Lahore, Pakistan. This article is an open access article distributed under the terms and conditions of the Creative Commons Attribution (CC-BY) license (http://creativecommons.org/licenses/by/4.0/). 